\title{
The Rho/Rac exchange factor Vav2 controls nitric oxide-dependent responses in mouse vascular smooth muscle cells
}

\author{
Vincent Sauzeau,1,2 María A. Sevilla, ${ }^{1,3}$ María J. Montero,1,3 and Xosé R. Bustelo1,2 \\ ${ }^{1}$ Centro de Investigación del Cáncer, 2Instituto de Biología Molecular y Celular del Cáncer, and Departamento de Fisiología y Farmacología, \\ CSIC-University of Salamanca, Campus Unamuno, Salamanca, Spain.
}

\begin{abstract}
The regulation of arterial contractility is essential for blood pressure control. The GTPase RhoA promotes vasoconstriction by modulating the cytoskeleton of vascular smooth muscle cells. Whether other Rho/Rac pathways contribute to blood pressure regulation remains unknown. By studying a hypertensive knockout mouse lacking the Rho/Rac activator Vav2, we have discovered a new signaling pathway involving Vav2, the GTPase Rac1, and the serine/threonine kinase Pak that contributes to nitric oxide-triggered blood vessel relaxation and normotensia. This pathway mediated the Pak-dependent inhibition of phosphodiesterase type 5, a process that favored RhoA inactivation and the subsequent depolymerization of the F-actin cytoskeleton in vascular smooth muscle cells. The inhibition of phosphodiesterase type 5 required its physical interaction with autophosphorylated Pak1 but, unexpectedly, occurred without detectable transphosphorylation events between those 2 proteins. The administration of phosphodiesterase type 5 inhibitors prevented the development of hypertension and cardiovascular disease in Vav2-deficient animals, demonstrating the involvement of this new pathway in blood pressure regulation. Taken together, these results unveil one cause of the cardiovascular phenotype of Vav2-knockout mice, identify a new Rac1/Pak1 signaling pathway, and provide a mechanistic framework for better understanding blood pressure control in physiological and pathological states.
\end{abstract}

\section{Introduction}

The Rho/Rac GTPase family is composed of a large number of members that, according to structural similarity criteria, are subclassified into Rho, Rac, and Cdc42 subfamilies (1). Upon activation, these GTPases bind to effectors responsible for triggering disparate biological responses, such as F-actin cytoskeletal changes, cell polarity, cell-cycle transitions, cytokinesis, and vesicle trafficking (1-3). These GTPase-dependent responses affect the behavior of single cells or, alternatively, promote coordinated multicellular events to educe responses at the whole-tissue level (1-3). The regulation of arterial contractility is one of the most conspicuous examples of multicellular responses triggered by Rho/Rac proteins $(2,4)$. Thus, it has been shown that numerous vasoconstrictors stimulate RhoA in local populations of vascular SMCs (vSMCs) that, in turn, promote the localized constriction of arterial blood vessels that leads to elevations in blood pressure $(2,4)$. This process is mediated by the induction of stress fibers and myosin contractility in stimulated vSMCs via the phosphorylation-dependent upregulation of the 20-kDa myosin light chain $\left(\mathrm{MLC}_{20}\right.$ ) and the downmodulation of its inhibitor, the $\mathrm{MLC}_{20}$ phosphatase (MLCP). These phosphorylation events are mediated by the Rho-associated coiled-coil containing protein kinase 1 (Rock1) $(5,6)$, a well-known RhoA effector (7). When a vasorelaxation response is required, the RhoA/Rock pathway becomes inactivated in those vSMCs through the active engagement of additional stimuli and signaling routes. One of these stimuli is NO, a gas synthesized by vascular endothelial cells. NO binds and activates soluble guanylate cyclase (sGC) in vSMCs, leading to cyclic GMP (cGMP) production and stimulation of the cGMP-dependent protein kinase type I (cGKI) $(8,9)$. Stimulated

Conflict of interest: The authors have declared that no conflict of interest exists. Citation for this article: J. Clin. Invest. 120:315-330 (2010). doi:10.1172/JCI38356.
cGKI triggers the phosphorylation of RhoA on serine 118, a posttranslational modification that induces the release of the GTPase from the plasma membrane and the inactivation of its downstream routes (10). NO also contributes to vasorelaxation by decreasing the activity of $\mathrm{MLC}_{20}$ kinase (MLCK), an $\mathrm{MLC}_{20}$ activator (11). The signal output of the NO-dependent pathway is further fine-tuned by the action of phosphodiesterase type 5 (PDE5), an enzyme that antagonizes the activation of the sGC pathway by hydrolyzing cGMP (12). Experimental and clinical evidence indicate that NOand RhoA-dependent pathways are essential to ensure normotensia and to adapt blood pressure rates to fluctuating environmental and physiological conditions. Thus, it has been shown that the inactivation of endothelial NO synthase and cGKI by genetic means leads to hypertension $(8,13-15)$. Moreover, PDE5 and Rock inhibitors restore defective vasodilatation responses associated with diverse cardiovascular pathologies (4, 7, 16-18).

Given the important role of these GTPases in modulating F-actin dynamics (1-3), it is possible that additional Rho/Rac proteins and Rho/Rac regulators could contribute to blood pressure control. Two candidates for such a role are Vav2 and Vav3, 2 tyrosine-phosphorylation-dependent enzymes that catalyze the exchange of guanosine nucleotides on Rho/Rac proteins (19-23). Such activity favors the transition of the GTPase substrates from the inactive (GDP bound) to the active, GTP-bound state (19-23). We have recently shown that mice lacking either Vav2 or Vav3 develop hypertension, cardiovascular remodeling, heart/kidney fibrosis, and renal dysfunctions, suggesting an implication of those GTPase upstream regulators in the control of blood normotensia in vivo $(24,25)$. Although it has been demonstrated that this cardiovascular phenotype is dependent on the activation of the renin/angiotensin II route $(24,25)$, the signaling defects responsible for those pathophysiological alterations remain unknown. 

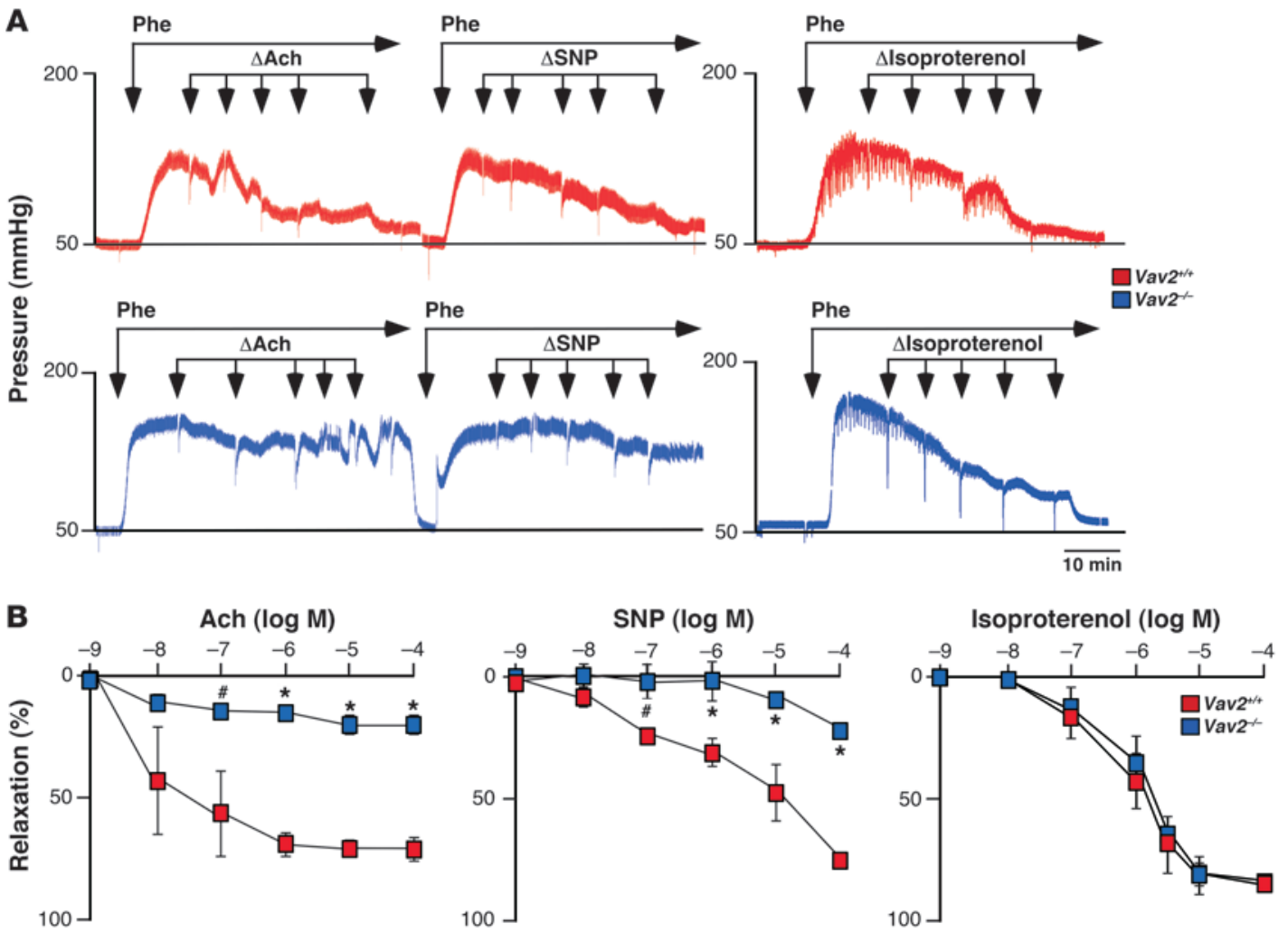

Figure 1

Defective vasodilatation responses of renal arteries from Vav2-/- mice to NO. (A) Examples of real-time recordings of the response of renal arteries from the indicated mouse strains to either the single or the sequential administration (arrows) of Phe, acetylcholine (Ach), SNP, and isoproterenol. Scale bar: 10 minutes. (B) Percentage of vessel relaxation induced by the indicated doses of acetylcholine, SNP, and isoproterenol on Phe-constricted renal arteries $(n=3-7)$. ${ }^{\#} P<0.05$; ${ }^{*} P<0.01$ compared with wild-type controls. log M, logarithm of the molar concentration used for each agent. Data are shown as mean \pm SEM.

To tackle this question, we have aimed at identifying the defective signal transduction pathways present in Vav2 $2^{-/-}$mice in the present work. Here, we report the discovery of a new NO- and Vav2-dependent crosstalk between Rac1 and RhoA GTPases that is essential for the regulation of blood vessel resistance and, when deregulated, to the establishment of a hypertensive state. In addition to shedding light onto the ontology of the hypertension and renocardiovascular disease of Vav2-deficient mice, these results identify unexpected new signaling elements and crosstalk of the Vav2/Rac1 route, suggest new roles for this pathway in signaling diversification and amplification events, and perhaps more importantly, provide a better understanding of the signaling routes controlling arterial contractility and blood pressure rates.

\section{Results}

Vav2 deficiency leads to ineffective NO-dependent vasodilatation. To identify the cause(s) of the hypertension of $\mathrm{Vav}^{-/-}$mice, we first examined the reactivity of their blood vessels to a variety of stimuli. We surmised a potential role of Vav2 in this biological response, since this exchange factor displayed higher expression levels than the rest of Vav family members in both conductance and resistance arteries (Supplemental Figure 1; supplemental material available online with this article; doi:10.1172/JCI38356DS1). We observed that renal arteries obtained from Vav2-deficient mice reacted as their wild-type counterparts to vasoconstrictor agents such as the $\alpha 1$-adrenergic receptor agonist phenylephrine (Figure $1 \mathrm{~A}$ and Supplemental Figure 2A), $\mathrm{K}^{+}$(Supplemental Figure 2A), or angiotensin II (144.29 $\pm 4.43[n=7]$ versus $150.50 \pm 17.40[n=6]$, respectively; see also Supplemental Figure 14). However, we found that the subsequent relaxation response induced by acetylcholine was impaired in the case of $\mathrm{Vav}^{-/-}$animals (Figure 1, A and B). Acetylcholine promotes vasodilatation by inducing the synthesis of NO by vascular endothelial cells (26). The same defective response was observed when we treated phenylephrine-constricted renal arteries with the NO donor sodium nitroprusside (SNP) (Figure $1, \mathrm{~A}$ and $\mathrm{B}$ ), indicating that the vasodilatation defect of the arteries from $\mathrm{Vav}^{-1-}$ mice was due to dysfunctional responses of vSMCs to NO rather than to defective NO production. Instead, we recorded normal relaxation responses when renal arteries of those mice were stimulated with isoproterenol (Figure 1, A and B), a $\beta 1 / \beta 3$-adrenergic receptor agonist that promotes vasodilatation through the stimulation of cAMP-dependent routes in vSMCs and the production of vasoconstrictor agents such as prostacyclin and endothelium-derived hyperpolarizing factor (EDHF) in endothelial cells. These results indicated that the poor reactivity to NO-dependent signals was not due to either a general defect in the mechanoelastic properties of $\mathrm{Vav}^{-/-}$arteries or to a general dysfunction in endothelial or vSMCs reactivity to vasodilatation stimuli. Two independent analyses confirmed that the reactivity problems of $\mathrm{Vav2}^{-/-}$resistance arterioles were not an indirect con- 
A
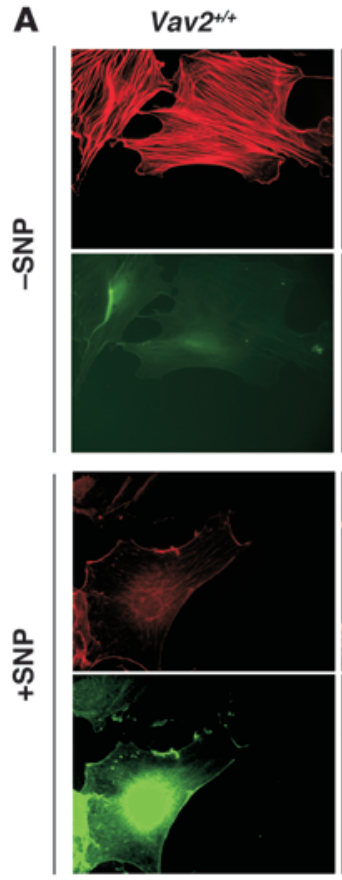

Vav2 $^{-1-}$

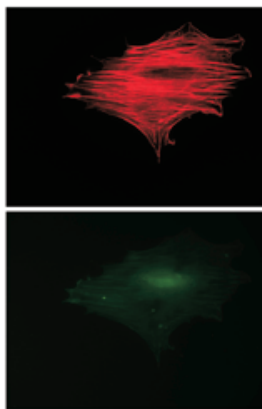

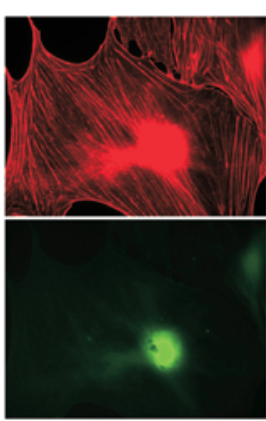

$\operatorname{Vav3}^{--}$
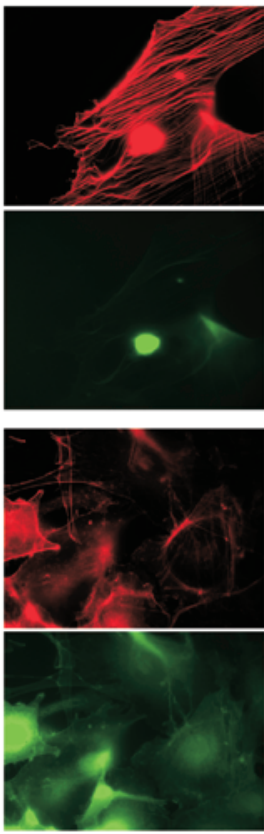

C

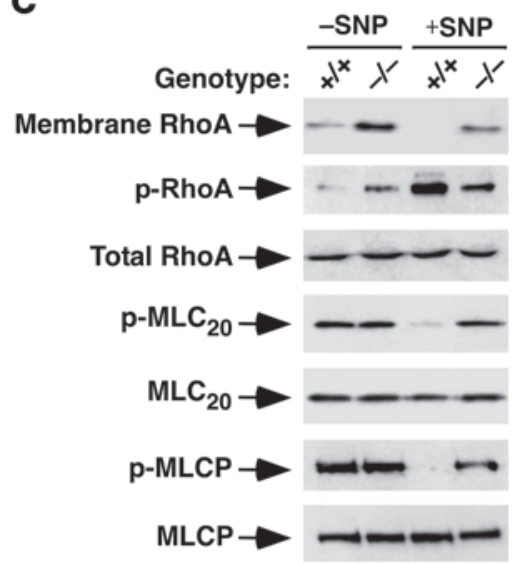

D

D
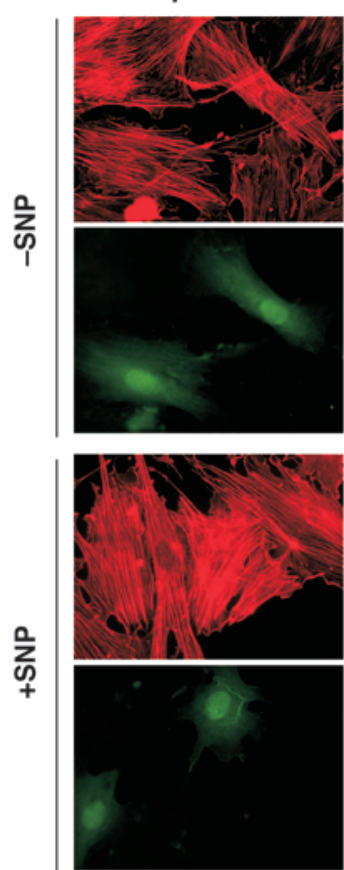

Vav2 $2^{--} ;$Vav3 $^{--}$
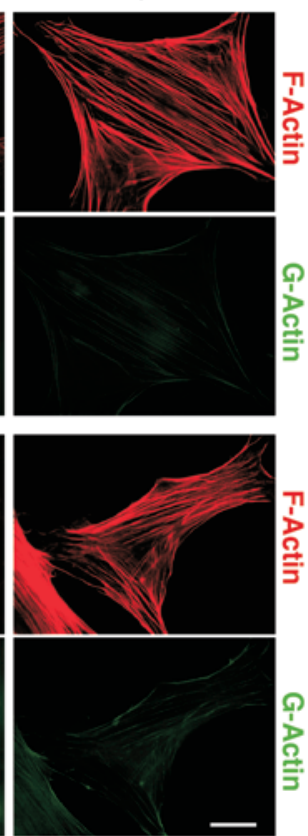

B

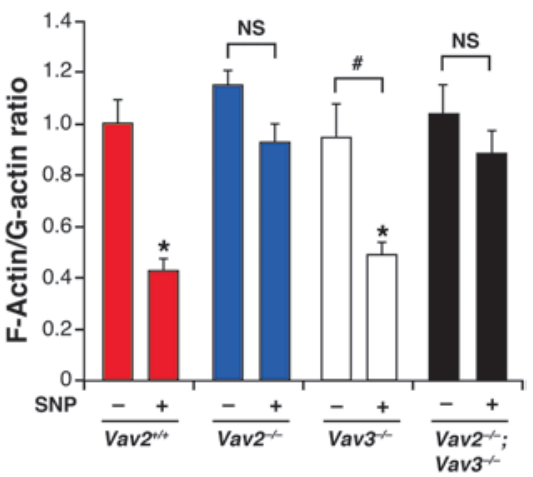

Experimental condition
E
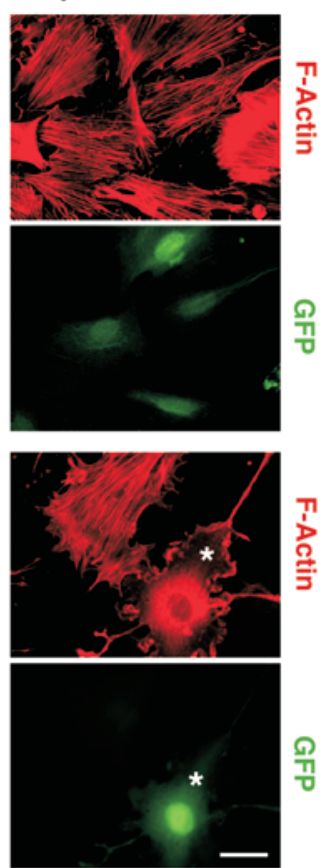

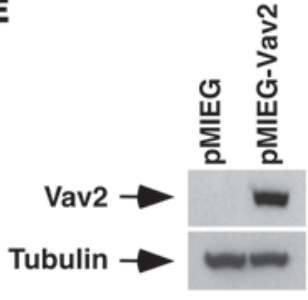

$\mathbf{F}$

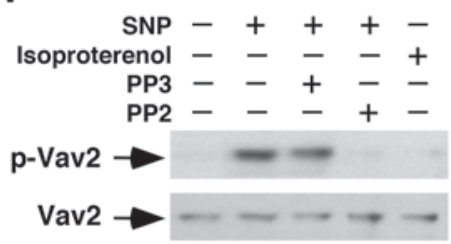

Figure 2

Vav2 is essential for NO-triggered F-actin depolymerization and Rho/Rock pathway inactivation in vSMCs. (A) Representative images of F-actin (red) and G-actin (green) in vSMCs of the indicated genotypes that were treated (+) or untreated (-) with SNP for 1 hour as described. Scale bar: $20 \mu \mathrm{m}$. (B) F-actin/G-actin ratio in vSMCs of the indicated genotypes before $(-)$ and after $(+)$ SNP stimulation $(n \geq 4)$. ${ }^{*} P<0.05$; ${ }^{*} P<0.01$ compared with either nonstimulated wild-type cells (which were given an arbitrary value of 1 ) or the indicated nonstimulated cells (brackets). NS, values not statistically significant. Data are shown as mean + SEM. (C) Top panel: membrane localization of RhoA in wild-type and Vav2-IvSMCs before (-) and after (+) SNP stimulation for 1 hour. Remainder of panels: phosphorylation and total expression levels of the indicated proteins in vSMCs before and after SNP stimulation. p-, phospho-protein. (D) Vav2 ${ }^{-/-}$vSMCs were infected with the indicated retroviruses and left untreated $(-)$ or treated $(+)$ with SNP for 1 hour as shown; their F-actin cytoskeleton was visualized by fluorescence microscopy. F-actin and GFP-derived fluorescence signals are shown in red and green, respectively. Scale bar: $20 \mu \mathrm{m}$. *, an EGFP-positive cell showing F-actin depolymerization upon SNP stimulation. (E) Anti-Vav2 (top panel) and antitubulin (bottom panel) immunoblot analysis of total cellular lysates obtained from the indicated retroviral infections of Vav2 ${ }^{--}$VSMCs. (F) Antiphosphotyrosine (top panel) and anti-Vav2 (bottom panel) immunoblot analysis of anti-Vav2 immunoprecipitates obtained from lysates of wild-type vSMCs cultured under the indicated conditions. 
A

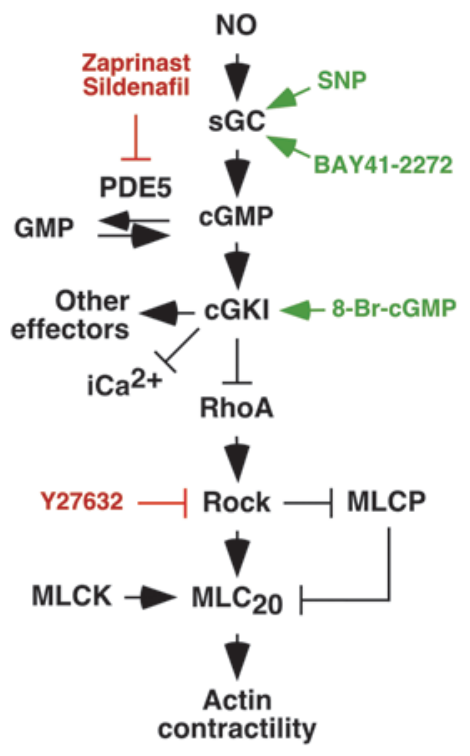

B
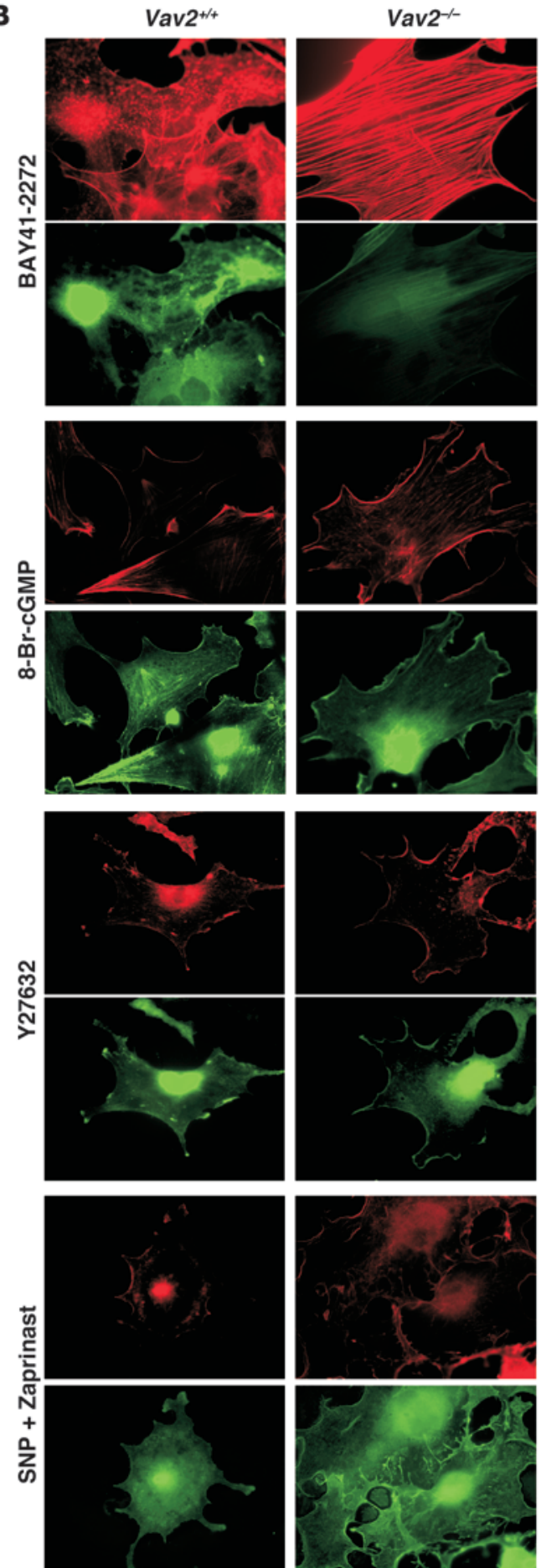
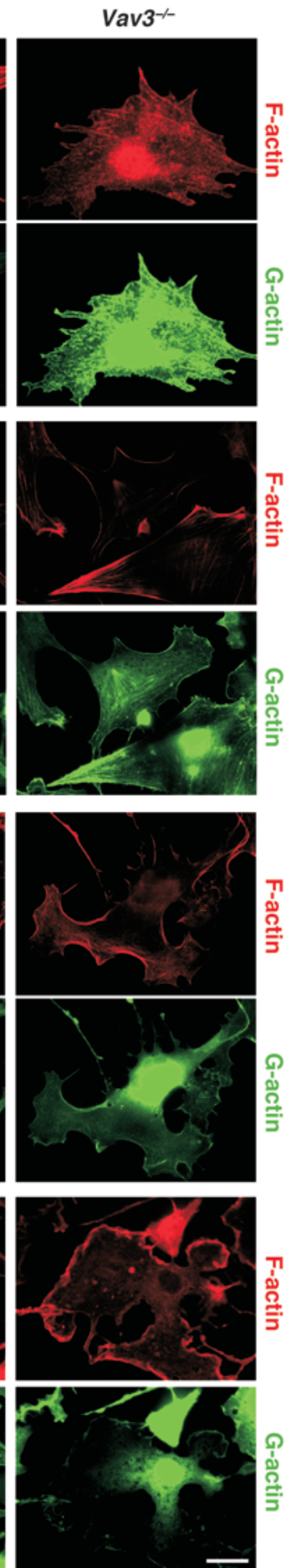

Figure 3

Defective regulation of PDE5 activity in Vav2-- vSMCs. (A) Schematic representation of the sGC pathway in vSMCs. Known agonist and antagonist molecules of elements of this route are shown in green and red, respectively. $\mathrm{iCa}^{2+}$, intracellular calcium. (B) Representative examples

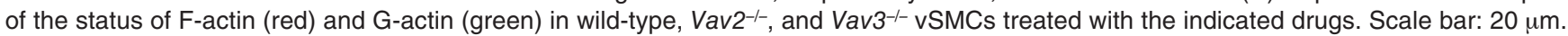
(C) F-actin/G-actin ratio obtained in the above experiments $(n \geq 3)$. ${ }^{*} P<0.01$ compared with nonstimulated wild-type cells (which received an arbitrary value of 1 ). Data are shown as mean + SEM. 

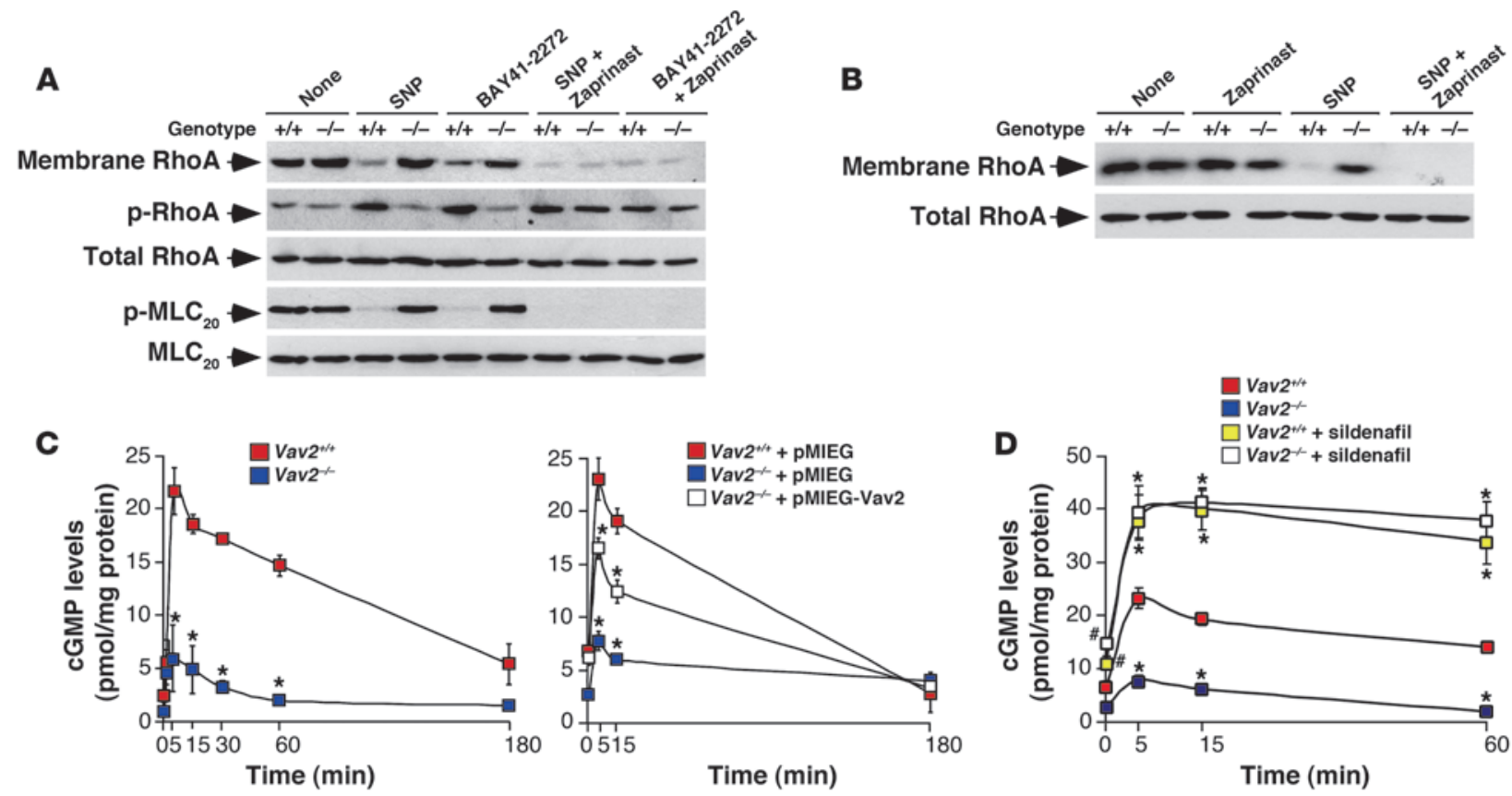

Figure 4

Defective regulation of PDE5 activity in Vav2 $2^{--}$vSMCs. (A and B) Top panels: membrane localization of RhoA in wild-type and Vav2 $2^{--}$vSMCs either untreated (none) or treated with the indicated drugs. Remainder of panels: phosphorylation and total expression levels of the indicated proteins in control and Vav2 ${ }^{--}$vSMCs after the above treatments. (C and D) Time course of cGMP production induced by SNP in the indicated primary vSMCs (C, left panel: $n=4$, each performed in triplicate), retrovirally-infected vSMCs (C, right panel: $n=2$, each performed in triplicate), and sildenafiltreated vSMCs (D: $n=2$, each performed in triplicate). ${ }^{\prime} P<0.05$; ${ }^{*} P<0.01$ compared with wild-type cells. Data are shown as mean \pm SEM.

sequence of the vascular remodeling occurring in those mice (24). Thus, the same defective response to SNP was found in renal arteries from Vav2 $2^{-/-}$animals at 1 month of age (Supplemental Figure 2B), an age in which those mice have not yet developed cardiovascular remodeling (24). In addition, we did not detect any reactivity problems to SNP in renal arteries obtained from 4-month-old Vav $3^{-/}$mice (Supplemental Figure 2C), a mouse strain that also develops hypertension and extensive vascular remodeling (25). Taken together, these results indicate that $\mathrm{Vav} 2^{-/-}$mice have intrinsic defects in the signaling response of vSMCs to NO.

Vav2 $2^{-1-} v$ SMCs show defective inactivation of the RhoA/Rock pathway. Since NO promotes the disassembly of the vSMC F-actin cytoskeleton via inactivation of the RhoA/Rock pathway (10), we evaluated the ability of SNP to induce such biological response in primary cultures of $\mathrm{Vav}^{-/-}$vSMCs. As a control, we used vSMCs from both wild-type and $\mathrm{Vav}^{-/-}$mice. We observed that SNP triggered a defective disassembly of stress fibers in Vav2 ${ }^{-/-}$vSMCs when compared with wild-type and Vav3 ${ }^{-/-}$cells (Figure 2, A and B). Similarly, SNP did not promote effective cytoskeletal responses in vSMCs derived from a knockout mouse strain lacking both Vav2 and Vav3 (Figure 2, A and B) (24). The lack of proper stress fiber disruption in $\operatorname{Vav}^{-{ }^{--}}$vSMCs was linked to improper inactivation of the RhoA/Rock pathway because those cells showed marked defects in RhoA phosphorylation, RhoA removal from membranes, and the dephosphorylation of both MLCP and $\mathrm{MLC}_{20}$ upon SNP stimulation (Figure $2 \mathrm{C}$ ). The responsiveness of primary Vav2 $2^{-/}$vSMCs to SNP was rescued by retroviruses expressing bicistronically wild-type Vav2 and the GFP but not by retroviruses encoding GFP alone (Figure 2, D and E).
Given that Vav2 becomes activated by tyrosine phosphorylation $(20,27)$, we surmised that the engagement of its pathway by NO had to entail tyrosine phosphorylation events. To verify this possibility, we immunoprecipitated the endogenous Vav2 protein from nonstimulated and NO-stimulated vSMCs and measured its phosphorylation status using Western blot analysis with anti-phosphotyrosine antibodies. These analyses indicated that SNP induces the phosphorylation of this exchange factor on tyrosine residues (Figure 2F). This phosphorylation event was probably mediated by Src family proteins because it could be inhibited by a Src-specific inhibitor (PP2) (Figure 2F). A control molecule that does not inhibit Src kinases (PP3) did not affect Vav2 phosphorylation levels (Figure 2F). Activation of Src family proteins by $\mathrm{NO}$ has been shown before (28). The NO/Src-dependent phosphorylation of Vav2 was specific for the NO signaling pathway because we could not detect Vav2 phosphorylation in isoprotrenol-stimulated wildtype vSMCs (Figure 2F). Taken together, these results indicate that Vav2 is an integral component of the NO pathway in vSMCs.

Vav2-/- $v$ SMCs show defective regulation of PDE5 and reduced cGMP production. Immunoblot (Figure 2C) and RT-PCR (Supplemental Figure 3) analyses demonstrated that the expression levels of the main regulatory elements involved in the NO pathway were not affected in the absence of Vav2. To pinpoint the signaling defect of Vav2-deficient vSMCs, we performed rescue experiments with agonist molecules for sGC (BAY41-2272) and cGKI (8-Br-cGMP) as well as with inhibitors for Rock (Y27632) (Figure 3A). The addition of 8-Br-cGMP and Y27632 induced F-actin depolymerization in the absence of SNP stimulation regardless of the genotype of the vSMCs used (Figure 3, B and C), indicating that the signaling route 


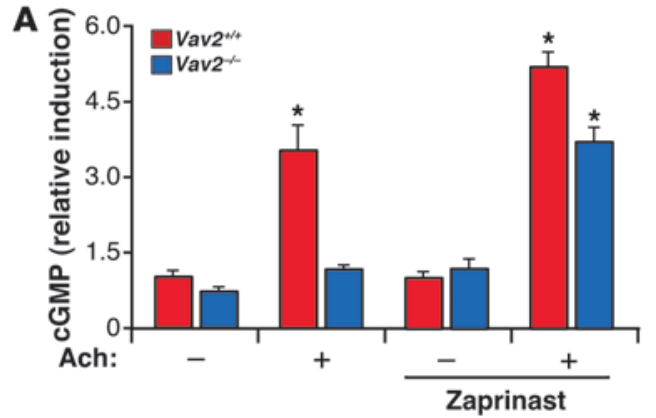

B
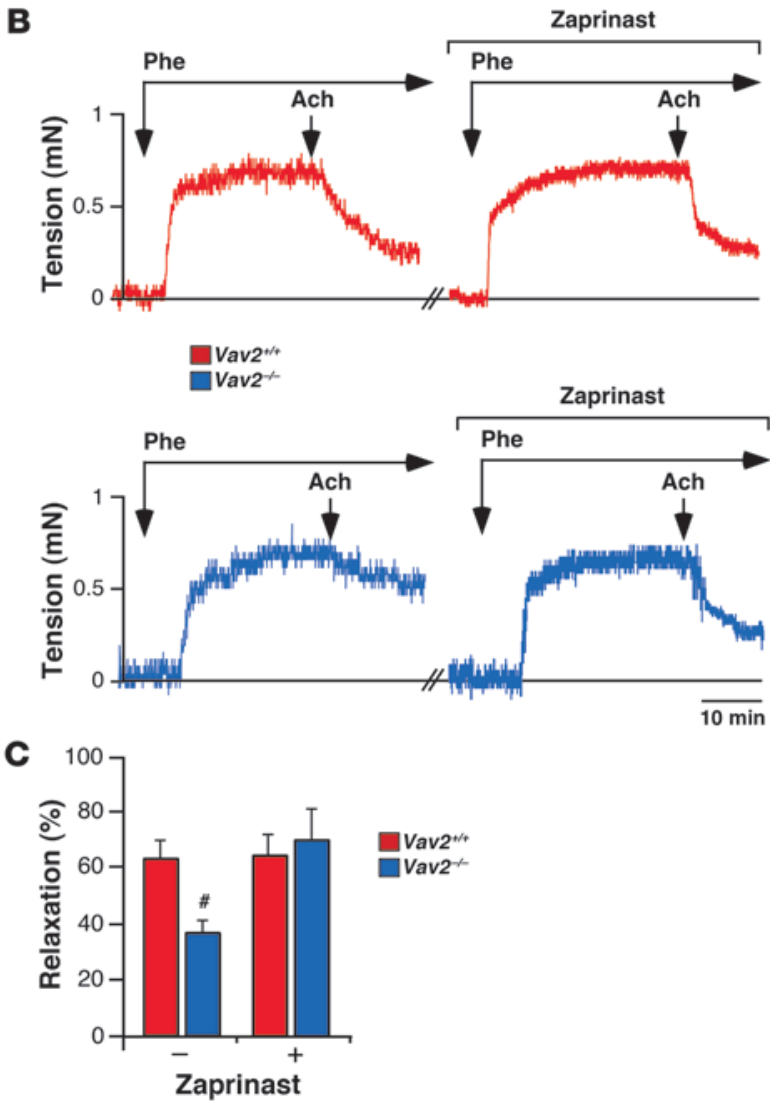

downstream of cGKI was not dysfunctional in Vav2 $2^{-/-}$mice. In contrast, BAY41-2272 induced the disassembly of stress fibers in wildtype and Vav3 ${ }^{---}$vSMCs but not in Vav2-deficient cells (Figure 3, B and C). This compound was also ineffective at promoting RhoA phosphorylation, the release of RhoA from membranes, and the dephosphorylation of $\mathrm{MLC}_{20}$ in $\mathrm{Vav}^{-/-}$vSMCs (Figure 4A). These results indicate that the signaling defect present in Vav2-deficient vSMCs was placed downstream of sGC but upstream of cGKI.

Since the only known enzyme working at that level is PDE5 (Figure $3 \mathrm{~A}$ ), we next investigated the functional status of this enzyme in Vav2 $2^{-/-}$mice. Indirect observations suggested that this enzyme was hyperactive in those cells because a PDE5 inhibitor (zaprinast) (Figure 3A) rescued the responsiveness of Vav2-deficient cells to SNP (Figure 3, B and C, and Figure 4, A and B) and BAY41-2272 (Figure 4A). As a control, we observed that zaprinast had no significant effects on the F-actin cytoskeleton (Figure 3C) and the amount of membrane-bound RhoA (Figure 4B) when added alone to nonstimulated wild-type and Vav2 ${ }^{-/-}$vSMCs. PDE5 seems to be

\section{Figure 5}

Defective vasodilatation responses of aortas from Vav2 $^{-/-}$mice to NO. (A) Production of cGMP in control and zaprinast-treated aortas from the indicated mouse strains after acetylcholine (Ach) administration $(n=3) .{ }^{*} P<0.01$ compared with the appropriate wild-type control. (B) Examples of real-time recordings of the response of aortas from the indicated mouse strains to the serial administration (arrows) of phenylephrine (Phe, $1 \mu \mathrm{M})$ and acetylcholine $(1 \mu \mathrm{M})$. To inhibit PDE5 activity, aortas were incubated when indicated with $10 \mu \mathrm{M}$ zaprinast 30 minutes before the administration of phenylephrine. Scale bar: 10 minutes. (C) Percentage of vessel relaxation induced by acetylcholine on phenylephrine-constricted aortas $(n=4-5)$. ${ }^{\#} P<0.05$ compared with wild-type controls. Data are shown as mean + SEM.

the only PDE family member involved because the treatment of Vav2 $2^{-/}$vSMCs with either PDE1 (vinpocetine) or PDE2 (erythro9-[2-hydroxy-3-nonyl]adenine [EHNA]) inhibitors did not eliminate their defective cytoskeletal responses to SNP (Supplemental Figure 4). The regulation of PDE5 does not seem to involve significant membrane translocation/releasing events, since we have not detected PDE5 in membrane fractions independently of the experimental condition or the vSMCs genotype in these studies (Supplemental Figure 5).

Consistent with an implication of PDE5 in the Vav2 signal transduction pathway, we observed that the production of cGMP induced by SNP was severely compromised in Vav2 ${ }^{-/-}$vSMCs (Figure 4, C and D). This defect was rescued by overexpressing wildtype Vav2 in Vav2 ${ }^{-/-}$vSMCs (Figure 4C) or, alternatively, by treating those cells with the PDE5 inhibitor sildenafil (Figures 3A and 4D). The comparable levels of cGMP production observed in Figure 4D in all sildenafil-treated cells further confirmed that the reduced cGMP levels detected in NO-stimulated Vav2 $2^{-/-}$vSMCs were due to abnormal catabolic rates rather than to ineffective biosynthesis of this second messenger. To extrapolate these results to a more physiological setting, we first compared the cGMP levels in acetylcholine-stimulated aortas from wild-type and Vav2-deficient mice. As shown in Figure 5A, acetylcholine triggered significantly lower cGMP levels in the vasoconstricted aortas from $\mathrm{Vav2}^{-/-}$animals than in those obtained from wild-type controls. However, and consistent with our in vitro culture studies (see Figures 3 and 4), this signaling defect was overcome by the preincubation of aortas with zaprinast (Figure 5A). As expected, the low cGMP levels were associated with reduced vasodilatation responses of $\mathrm{Vav}^{-{ }^{-/}}$aortas to acetylcholine, a defect that was also eliminated by zaprinast (Figure 5, B and C). This signaling problem was also present in renal arteries from $\operatorname{Vav}^{-/-}$mice, because we observed normal vasodilatation responses to a cGMP analogue (8-pCPT-cGMP) (Figure 6, A and $\mathrm{B})$, or when the stimulation with acetylcholine was conducted in the presence of zaprinast (Figure 6C). Collectively, these results unveil a signaling link among NO, Vav2, and PDE5 in vSMCs. In addition, they show that Vav2 gene deficiency leads to an elevated, PDE5-dependent cGMP catabolism in purified vSMCs, aortas, and renal arteries.

Rac1 and Pak1 are involved in PDE5 inbibition in vSMCs. The main known function of Vav2 is to act as a GDP/GTP exchange factor for Rho/Rac GTPases $(20,27,29)$. Experiments conducted in primary vSMCs indicated that Rac1 was probably the Rho/Rac family member working downstream of Vav2 in those cells (see Supplemental Text and Supplemental Figures 6 and 7). The implication of Rho/Rac family GTPases in this response was further assessed by 


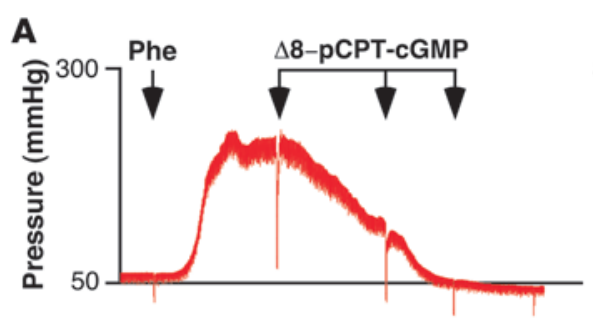

B
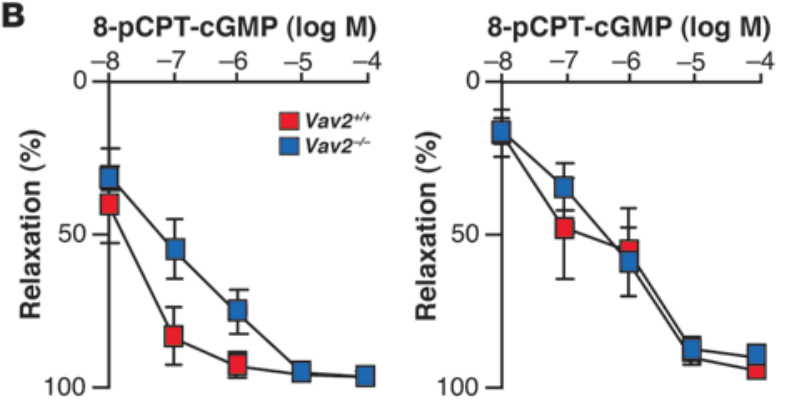

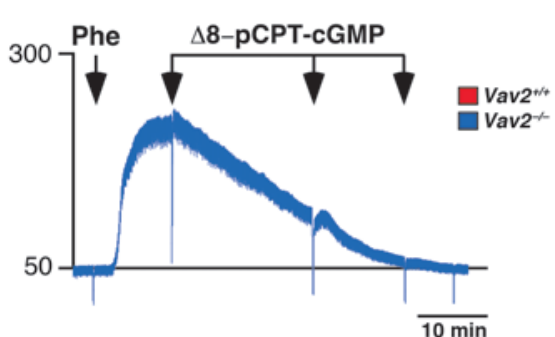

C
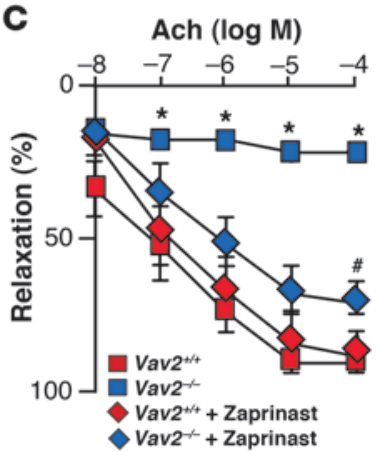

Figure 6

Addition of a cGMP analogue induces normal vasodilatation responses in Vav2-1- renal arteries. (A) Real-time recordings of the response of phenylephrine-constricted renal arteries from the indicated mouse strains to the sequential administration of 8-pCPT-cGMP. Scale bar: 10 minutes. (B) Percentage of vessel relaxation induced by the indicated doses of 8-pCPT-cGMP on phenylephrine-constricted renal arteries from 4-month-old (left panel; $n=5-6$ ) and 2-month-old (right panel; $n=3-6$ ) animals. (C) Percentage of relaxation induced by acetylcholine after phenylephrine administration in renal arteries from control and Vav2-knockout mice. When indicated, kidneys were perfused with $10 \mu \mathrm{M}$ zaprinast 30 minutes prior to the phenylephrine stimulation to inhibit PDE5. ${ }^{*} P<0.05$; ${ }^{*} P<0.01$ compared with wild-type controls. Data are shown as mean \pm SEM.

expressing Rac1 (F28L) and RhoA (F30L) mutants in vSMCs using a retrovirus system. These mutations generate GTPases with rapid GDP/GTP exchange cycles that result in enhanced GTP-loading rates and constitutive activation of downstream routes $(30,31)$. The ectopic expression of Rac1 ${ }^{\mathrm{F} 28 \mathrm{~L}}$ restored the cytoskeletal response of Vav2-deficient cells to SNP, leading to a disassembly of stress fibers comparable in intensity to that found in SNP-stimulated wild-type cells (Supplemental Figure 8). Instead, RhoA ${ }^{\mathrm{F} 30 \mathrm{~L}}$ blocked the disassembly of stress fibers in SNP-stimulated wild-type vSMCs (Supplemental Figure 8), hence reproducing the defects caused by the Vav2 deficiency in this cell type (see Supplemental Text for further information). To narrow down the Rac1 downstream effectors implicated in vSMC F-actin disassembly, we utilized 2 Rac1 ${ }^{\mathrm{F} 28 \mathrm{~L}}$ effector domain mutants that cannot engage specific downstream elements of the Rac1 pathway. Rac1 $1^{\mathrm{F} 28 \mathrm{~L}+\mathrm{F} 37 \mathrm{~A}}$ activates $\mathrm{p} 21^{\mathrm{Cdc} 42 / \mathrm{Rac} 1}$-activated kinase (Pak) and JNK, but it does not bind partner of Rac1 (Por1). In contrast, Rac1 ${ }^{\mathrm{F} 28 \mathrm{~L}+\mathrm{Y} 40 \mathrm{C}}$ does not activate Pak and JNK but can bind Por1 (32). We observed that the retroviral-mediated expression of Rac1 ${ }^{\mathrm{F} 28 \mathrm{~L}+\mathrm{F} 37 \mathrm{~A}}$ induced biological responses indistinguishable from those elicited by Rac1 $1^{\mathrm{F} 28 \mathrm{~L}}$ both in wild-type and Vav2 ${ }^{---}$vSMCs (Figure 7, A and B). Instead, Rac1 ${ }^{\mathrm{F} 28 \mathrm{~L}+\mathrm{Y} 40 \mathrm{C}}$ could not restore the cytoskeletal response of $\mathrm{Vav}^{-/-}$vSMCs to SNP and, in fact, it even blocked the F-actin depolymerization induced by SNP in wild-type vSMCs (Figure 7, A and B), suggesting that this protein may work as a dominant negative mutant for the entire Vav2/Rac1 pathway in this cell type. Importantly, Rac1 ${ }^{\mathrm{F} 28 \mathrm{~L}}$, but not Rac1 ${ }^{\mathrm{F} 28 \mathrm{~L}+\mathrm{Y} 40 \mathrm{C}}$, could also restore cGMP production in SNP-stimulated Vav2-/- vSMCs results indicate that the inactivation of Pak1 phenocopies the Vav2 deficiency in NO-stimulated vSMCs. Confirming the implication of Pak1 in this pathway, we demonstrated that the overexpression of Pak 1 restored normal cGMP production levels in Vav2 ${ }^{-/-}$vSMCs (Figure 8E).

Pak1 inhibits PDE5 enzyme activity in a kinase-independent manner. To shed light into the inhibitory mechanism of PDE5 activity by Pak1, we determined whether these 2 proteins could interact in vivo. We observed low levels of association between these proteins in nonstimulated wild-type and Vav2 ${ }^{-/-}$vSMCs (Figure 9A). The addition of SNP promoted a rapid and transient increase in such interaction in wild-type but not in Vav2 $2^{--}$vSMCs (Figure 9A). An SNP-dependent coimmunoprecipitation of PDE5 and Pak1 was also observed in aortas obtained from wild-type mice (Figure 9B). To identify the region of PDE5 involved in Pak1 binding, we generated glutathione-S-transferase (GST) fusion proteins containing PDE5 fragments (Figure 9C). After purification from E. coli (Figure 9D), we used pulldown experiments to test the association of those proteins with a Myc-tagged version of Pak1 ectopically expressed in COS1 cells. This strategy revealed that the PDE5 N terminus was involved in the physical interaction with Pak1 (Figure 9E). Using a similar approach with truncated fragments of Pak1 expressed in COS1 cells (Figure 9, F and G), we observed that the PDE5 N terminus recognized the Pak1 N-terminal region and not the Pak1 kinase domain (Figure 9G). The Nck, Grb2, and ArhGEF7 (a Rac1specific guanosine nucleotide exchange factor (GEF) also known as Pix and Cool) binding sites of Pak1 were also dispensable for the interaction with PDE5 (Figure 9, F and G). 


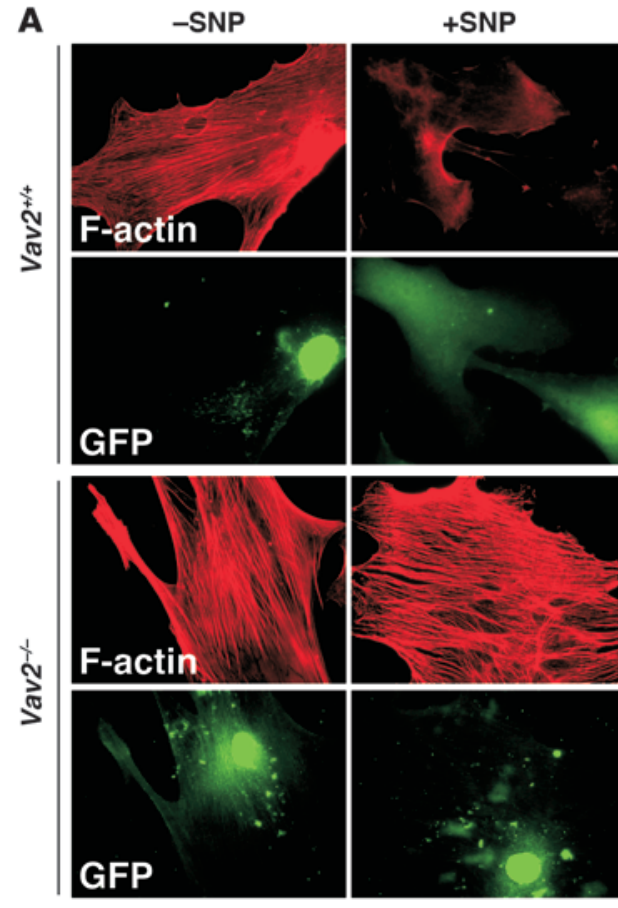

None
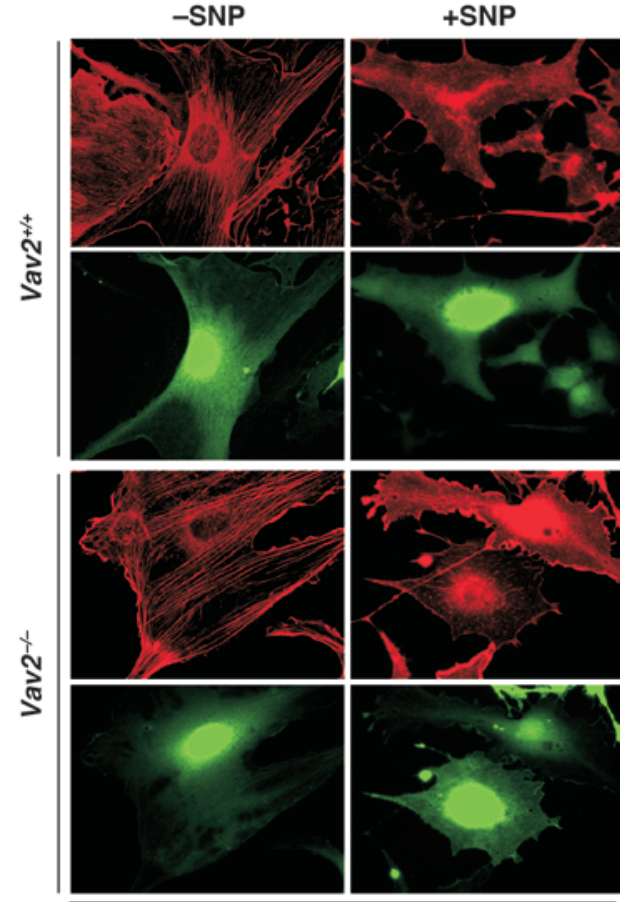

Rac1 ${ }^{\mathrm{F} 2 \mathrm{LL}+\mathrm{F} 37 \mathrm{~A}}$

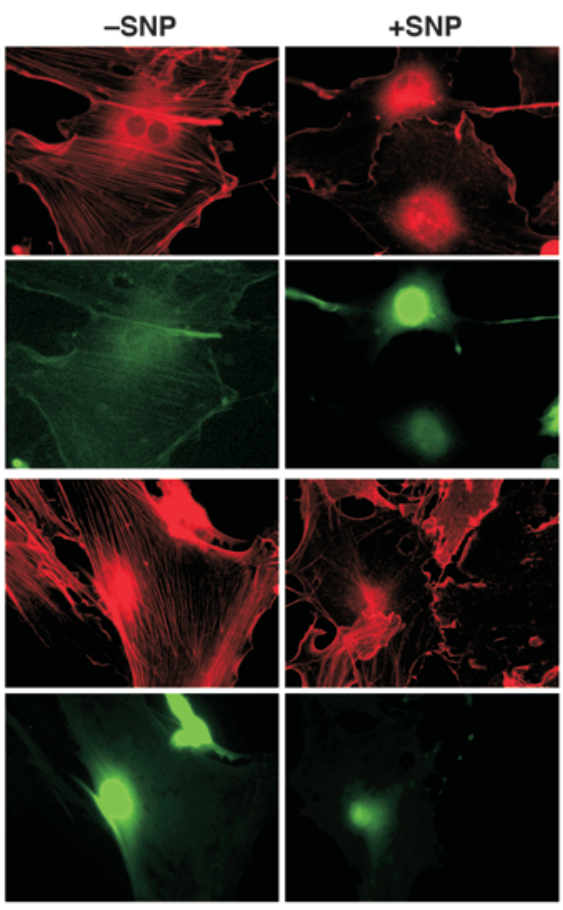

Rac1 ${ }^{\mathrm{F} 2 \mathrm{~L}}$
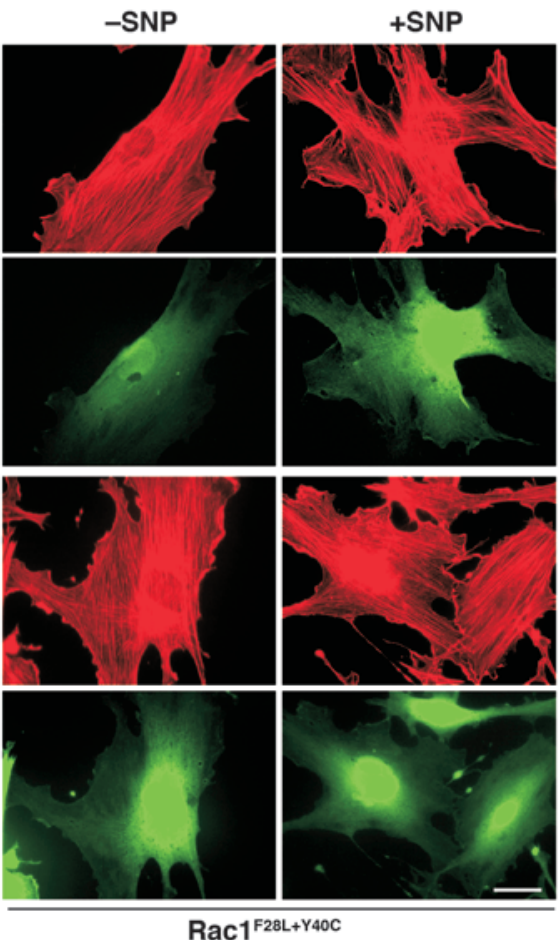

B

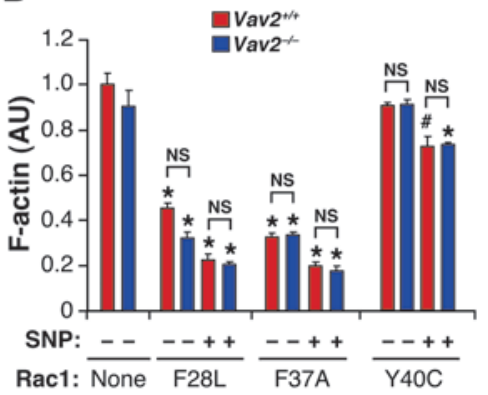

Experimental condition

Figure 7

Rac1 is involved in the NO-regulated Vav2 pathway. (A) Representative images of the F-actin cytoskeleton (red) present in nonstimulated (-) and SNP-stimulated (+) wild-type and Vav2 ${ }^{--}$vSMCs previously infected with retrovirus encoding bicistronically GFP (green) and the indicated Rac1 mutants. Scale bar: $20 \mu \mathrm{m}$. (B) F-actin levels obtained in the above experiments $(n=4)$. ${ }^{*} P<0.05$; ${ }^{*} P<0.01$; NS, not significant compared with either nonstimulated wild-type cells (which were given an arbitrary value of 1 ) or the indicated nonstimulated cells (brackets). Data are shown as mean + SEM. 
A
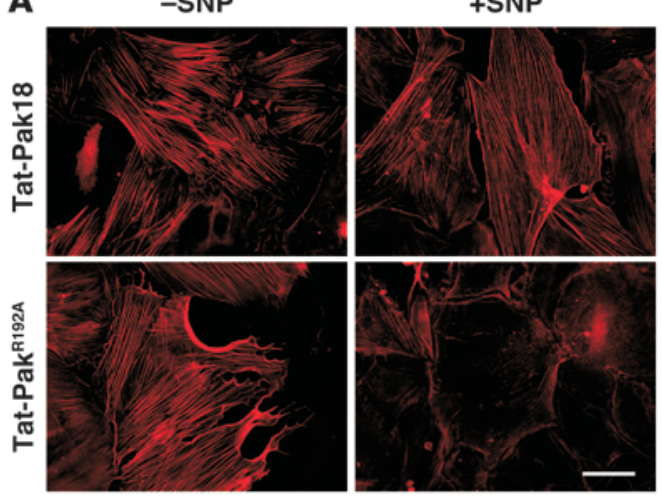

C

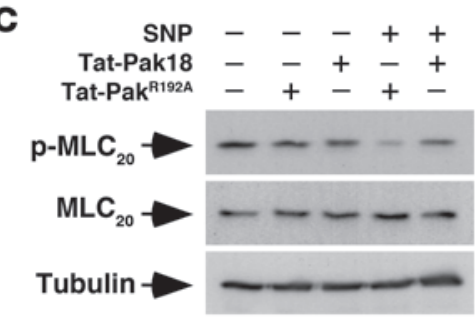

B

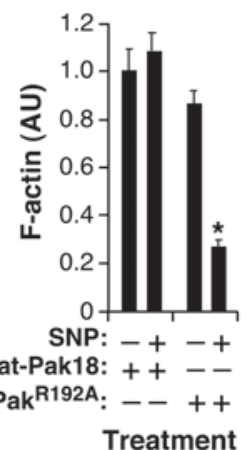

Treatment

\section{Figure 8}

Pak1 is involved in the NO-regulated Vav2 pathway. (A and B) Representative images (A) and quantitation (B) of the F-actin cytoskeleton present in wild-type vSMCs that were incubated with the indicated Tat peptides before SNP stimulation. Scale bar: $20 \mu \mathrm{m}$. ${ }^{*} P<0.01$ compared with wild-type cells. (C) Phosphorylation and total expression levels of $M^{2} C_{20}$ in wild-type vSMCs upon SNP stimulation under the indicated conditions. (D) F-actin levels of nonstimulated (-) and SNP-stimulated (+) wild-type vSMCs that were previously infected with either a control retrovirus or a retrovirus encoding the Pak1 $1^{\mathrm{K} 298 \mathrm{R}}$ dominant negative mutant $(n=4) .{ }^{*} P<0.01$ compared with wild-type cells. (E) Time course of cGMP production induced by SNP in the indicated cell type and retroviral infection conditions $\left(n=3\right.$, each performed in triplicate). ${ }^{*} P<0.01$ compared with mock-infected wild-type cells. Data are shown as mean \pm SEM.

Biochemical experiments indicated that Pak1 decreased the cGMP-hydrolyzing activity of PDE5 in an ATP-dependent manner (Figure 10A). This action was specific, because Pak1 had no effect on either the basal or the NO-stimulated activity of sGC (Supplemental Figure 11). Despite the ATP-dependent inhibitory effect of Pak1 on PDE5, we could not observe any significant phosphorylation of PDE5 by Pak1 using in vitro kinase reactions (Figure 10B). However, Pak1 could phosphorylate itself (Figure 10B) and transphosphorylate histone H1 (Figure 10B), demonstrating that we were using a catalytically active enzyme. Furthermore, the lack of PDE phosphorylation was not due to prior phosphorylation of the bacterial fusion proteins because we could phosphorylate the PDE5 N-terminal region and, to a lesser extent, the PDE5 catalytic domain using in vitro kinase reactions with PKA and cGKI $\alpha$ (Figure 10, C and D). These observations indicate that the need of ATP for PDE5 inhibition is probably due to Pak1 autophosphorylation rather than to Pak1-dependent PDE5 transphosphorylation. To further test this possibility, we investigated whether Pak1 autophosphorylation was required for its interaction with PDE5. We observed that whereas wild-type Pak1 interacted with the $\mathrm{N}$-terminal region of PDE5 in pulldown experiments, the catalytically impaired Pak1 $1^{\mathrm{K} 298 \mathrm{R}}$ mutant could not (Figure 10E). This result is in agreement with our previous in vivo data showing that Pak1 ${ }^{\mathrm{K} 298 \mathrm{R}}$ acts as a dominant negative mutant for the NO pathway in wild-type vSMCs (Figure 8D). These observations demonstrate that autophosphorylated Pak1 binds to the PDE5 N terminus and inhibits PDE5 catalytic activity through a phosphorylation-independent, protein/protein interaction event.

PDE5 inhibition blocks the bypertension and cardiovascular remodeling of Vav2-deficient mice. To assess whether the dysfunctions found in Vav2 $2^{--}$vSMCs contributed to the cardiovascular phenotype of Vav2-deficient mice, we evaluated the effect of the chronic administration of a PDE5 inhibitor on the evolution of the pathophysiological changes occurring in these animals. The oral administration of sildenafil for 3 months rescued the response of renal arteries to SNP (Supplemental Figure 12) and halted the development of hypertension (Figure 11A), vascular remodeling (Figure 11, B and C), hypertrophy of the left heart ventricle (Figure 11, B and C), and the increase in noradrenaline plasma levels (Supplemental Figure $13 \mathrm{~A}$ ) previously described in $\operatorname{Vav}^{-/-}$mice (24). Sildenafil also eliminated angiotensin II production (Figure 11A), a physiological event essential for the development of hypertension and cardiovascular disease in those mice (24). The responses elicited by sildenafil were similar to the effect of long-term treatments of these animals with captopril and propranolol (23). Sildenafil reduced, but did not abolish, the increase in adrenaline, aldosterone, and vasopressin levels typically present in Vav2 $2^{-/}$mice (Supplemental Figure 13A). The treatment with sildenafil was therefore less effec- 

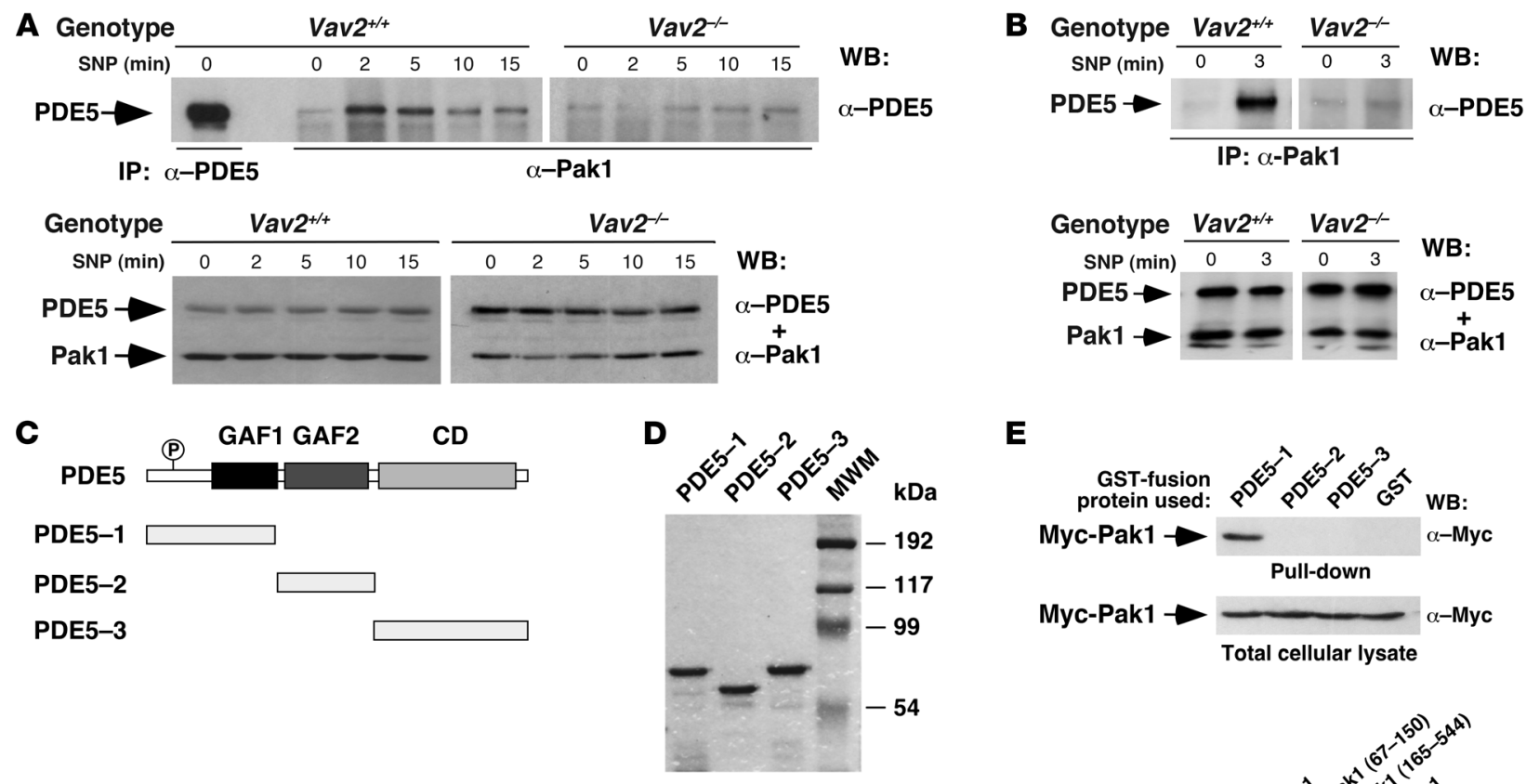

$\mathbf{E}$
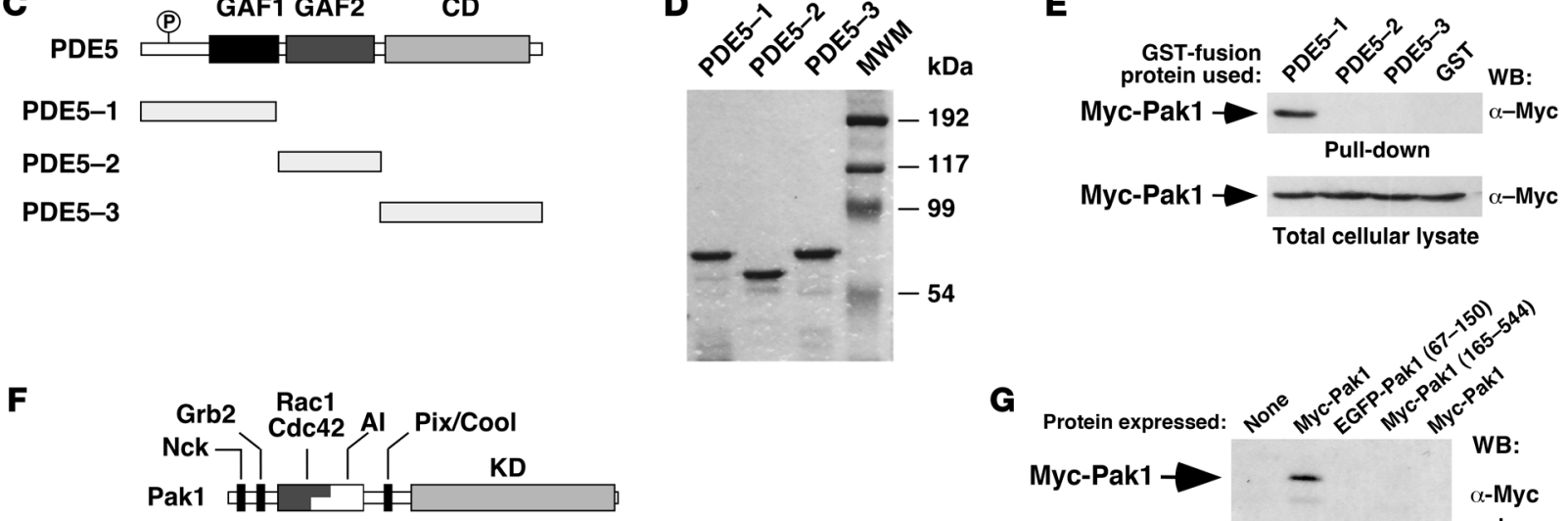

Pak1 (67-150)

Pak1 (165-544)
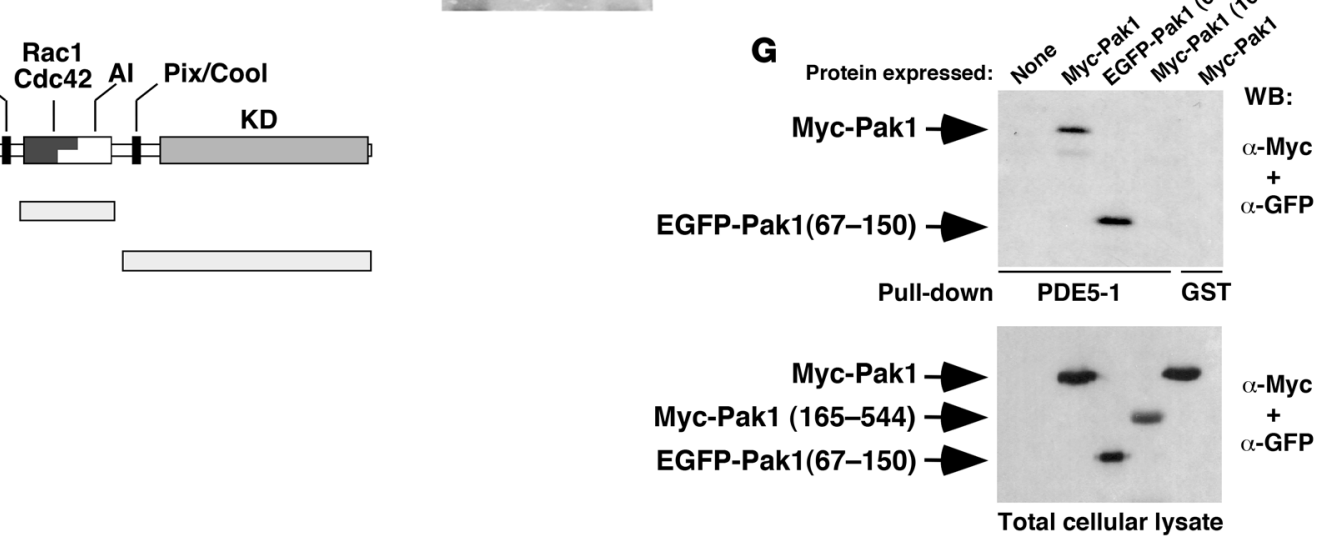

Figure 9

Physical interaction between Pak1 and PDE5. (A and B) Top panels: Detection by immunoblot of the presence of the endogenous PDE5 in antiPak1 immunoprecipitates obtained from either wild-type and Vav2 ${ }^{-/-}$VSMCs (A) or aortas (B) that were SNP stimulated for the indicated periods of time. An immunoprecipitate using anti-PDE5 antibodies was used as positive control in A. Bottom panels: Detection of endogenous PDE5 and Pak1 in the aliquots of the lysates used in the coimmunoprecipitation experiments by immunoblot analysis with the indicated combinations of antibodies. WB, western blot. (C) Schematic representation of PDE5 structure. The PDE5 regions used in the pulldown experiments are depicted below the full-length enzyme. P, phosphorylation site; GAF, domain present in cGMP-regulated cyclic nucleotide PDEs, adenyl cyclases, and the bacterial transcription factor FhIA; CD, catalytic domain. (D) SDS-PAGE analysis of aliquots of the GST-PDE5 fusion proteins after purification from E. coli. MWM, molecular weight markers. (E) Top panel: Immunoblot detection of wild-type Myc-Pak1 pulled down from COS1 cell lysates using the indicated GST proteins. Bottom panels: Immunoblot detection of Pak1 proteins in aliquots of the total cellular cell lysates used in the above experiments. (F) Schematic representation of Pak1. The binding sites for Nck, Grb2, Rac1/Cdc42, and Pix/Cool are indicated. Al, autoinhibitory motif; KD, kinase domain. The Pak1 fragments used in the pulldown experiments are indicated as gray bars underneath the full-length Pak1 protein. (G) Top panel: Immunoblot detection of wild-type Myc-Pak1 and/or the indicated Pak1 deletion mutants pulled down from COS1 cell lysates using the indicated GST proteins. Bottom panel: Immunoblot detection of Pak1 proteins in aliquots of the total cellular cell lysates used in the above experiments.

tive than propranolol in this response, since the administration of the latter drug could effectively reduce the concentrations of aldosterone and vasopressin found in the plasma of these animals to basal levels (23). Finally, we observed that the sildenafil treatment only affected marginally the tachycardia of Vav2 $2^{-/}$mice (Figure 11A) and did not eliminate the previously described hypertrophy of the adrenal gland medulla (data not shown). However, since this hypertrophy was established before the beginning of the sildenafil treatments (23), at this moment we cannot formally discern whether this lack of effect is due to the irreversibility of the hypertrophic state of adrenal chromaffin cells once it is established or to the independence of this phenotype from the blood vessel reactivity problems found in these animals.

To verify that the effects of sildenafil were related to the signaling deficiency of the Vav2/PDE5 route, we performed parallel experiments with Vav3-deficient mice, since these animals share many of the pathophysiological changes found in $\operatorname{Vav} 2^{-/-}$mice (25) without displaying any of their vascular NO reactivity problems (see above, 
A
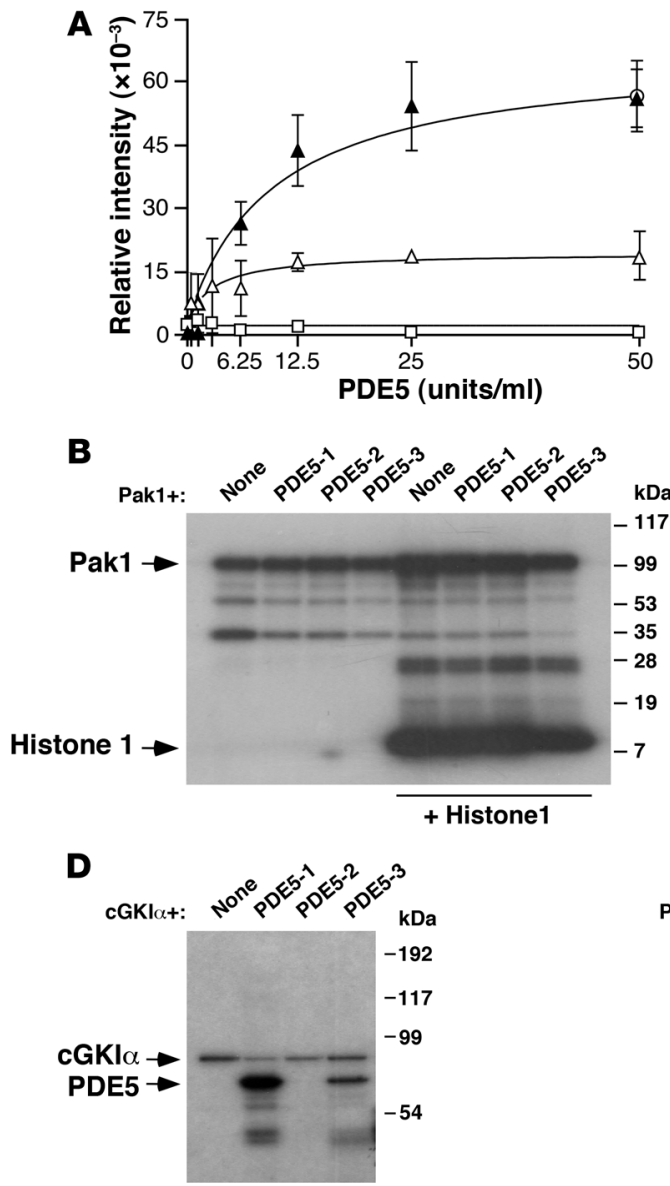

$\triangle$ PDE5

$\triangle$ Pak1 + PDE5 + ATP

Pak1 + PDE5 - ATP

口 Pak1

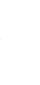
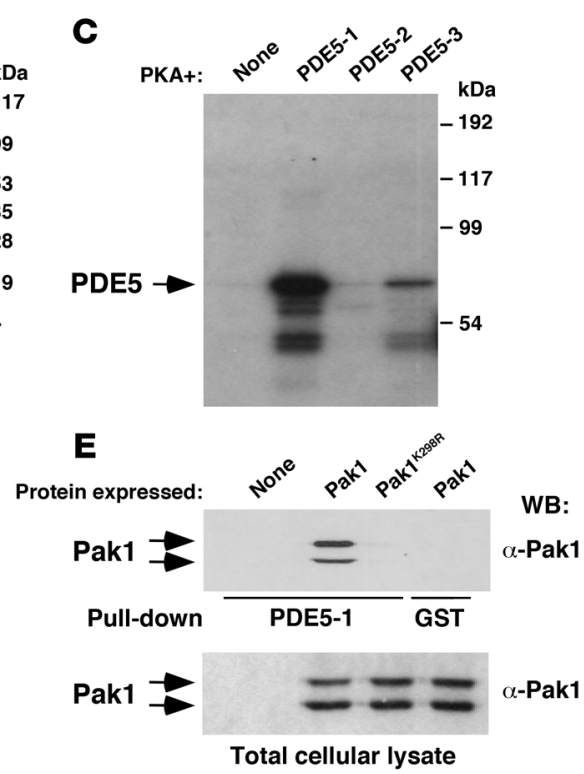

\section{Figure 10}

Pak1 inhibits PDE5 in a phosphorylationindependent manner. (A) In vitro PDE5 enzyme activity under the indicated conditions ( $n=4$, each performed in triplicate). Data are shown as mean \pm SEM. (B-D) The indicated GST-PDE5 fusion proteins (labeled as in Figure 9C) (B-D) alone or in combination with histone 1 (B) were subjected to in vitro kinase reactions in the presence of GST-Pak1 (B), PKA (C), and cGKI $\alpha$ (D). We also included reactions of kinases without PDE5 proteins (none, B-D). (E) The indicated Pak1 proteins (top) were expressed in COS1 cells and subjected to pulldown experiments with either the GST-PDE5-1 fusion protein (see Figure 9C) or the nonchimeric GST protein. The pulled down material (top panel) and aliquots of the total cellular lysates (bottom panel) used in the pulldown experiments were analyzed by anti-Pak1 immunoblot.
Supplemental Figure 2C and Figures 2 and 3). We found that sildenafil had no effect on the development of the hypertension of Vav3 $3^{-/}$mice (Figure 11A) and that it could not block the increase in angiotensin II (Figure 11A) and catecholamine (Supplemental Figure 13B) plasma levels present in these animals (25). In contrast, long-term treatments with captopril and propranolol did block angiotensin II and catecholamine production and, in addition, prevented the development of hypertension and associated cardiovascular remodeling in these animals (25). These results indicate that the rescue by PDE5 inhibitors of the cardiovascular defects present in Vav2 $2^{-/-}$mice is related to the specific signaling deficiency present in those animals and not due to a nonspecific action of those inhibitors in downstream physiological pathways that, like those activated by angiotensin II and catecholamines, contribute to the development of hypertension and cardiovascular remodeling. Consistent with this view, we also found that short-term treatments with zaprinast did not have any significant effect in the vasoconstriction response induced by angiotensin II in renal arteries from both wildtype and Vav2-knockout animals (Supplemental Figure 14). Taken together, these results confirm that the signaling defect related to improper PDE5 inactivation is a major contributing factor to the hypertensive and cardiovascular phenotype of Vav2-deficient mice.

\section{Discussion}

We have unveiled a new signal transduction pathway that ensures proper NO-dependent responses in vSMCs. This pathway is activated by the NO-dependent phosphorylation of Vav2 and leads to the sequential activation of Rac1 and Pak1. Activated Pak1 promotes the inhibition of PDE5 via the formation of a heteromolecular Pak1/PDE5 complex, thus favoring normal cGMP production, inactivation of the RhoA pathway through the cGKI-dependent phosphorylation of RhoA, and effective vasodilation (Figure 11D). To establish such interaction, Pak1 targets the PDE5 N terminus, a critical regulatory region that harbors the domains involved in homodimerization and the cGMP- and phosphorylation-dependent regulation of PDE5 activity (12). Unexpectedly, we have found that inactivation of PDE5 by Pak in vitro does not entail the phosphorylation of PDE5 on serine or threonine residues. However, unlike other biological responses previously reported for Pak kinases (34-38), such inhibitory activity cannot be categorized as kinase independent since it still requires ATP. Given that kinase-dead Pak1 mutants cannot interact physically with PDE5, these results suggest that Pak1 has to be phosphorylated to become accessible to the PDE5 $\mathrm{N}$ terminus. This is consistent with structural data indicating that Pak proteins become activated by the autophosphorylation-dependent disruption of inactive homodimers (38-40). Interestingly, the interaction of Pak1 with PDE5 occurs early and transiently during NO signaling, thus making this early inhibitory step compatible with the subsequent burst of PDE5 activity required for cGMP removal. Such transitory interaction also indicates that a feedback mechanism should be at work to disrupt the Pak1/PDE5 complex upon cGMP generation. Whether such feedback signals impinge on Pak1, on PDE5, or simultaneously on both proteins remains to be determined. 

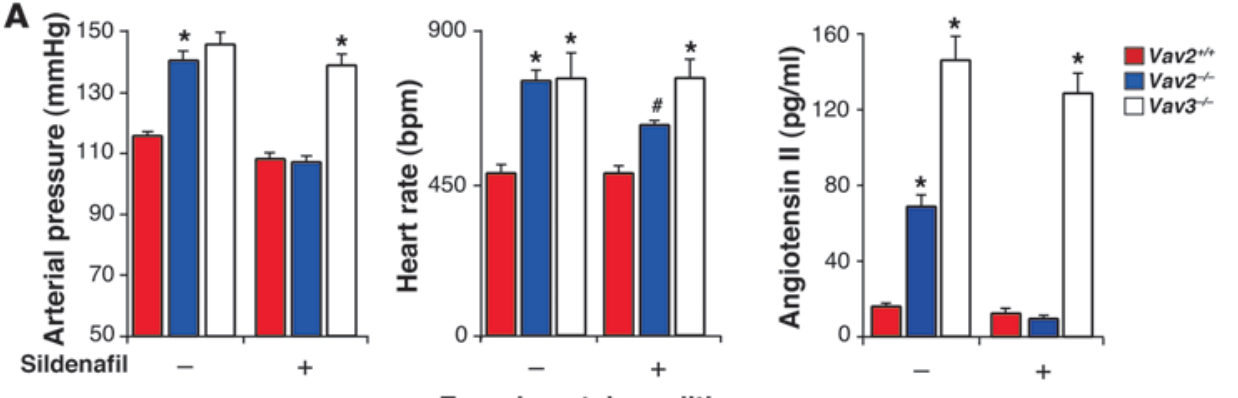

Experimental condition

B
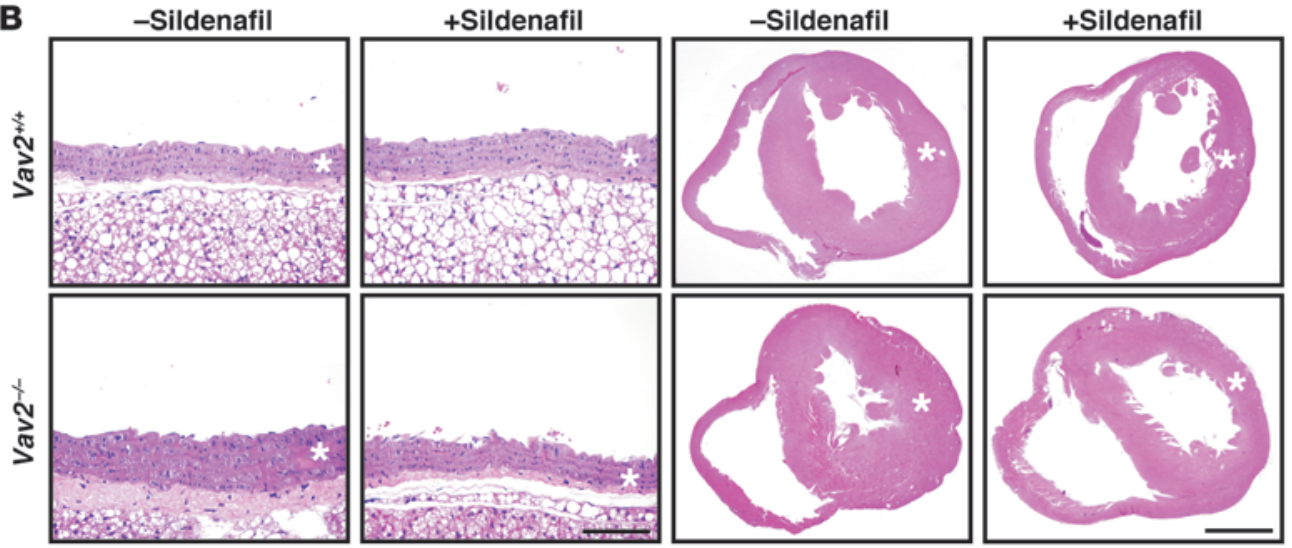

C

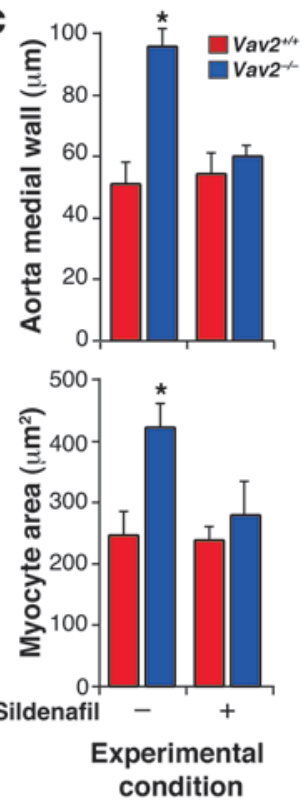

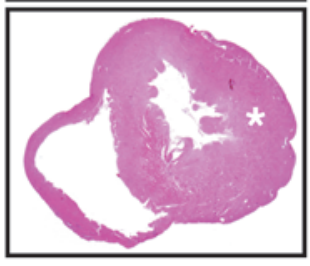

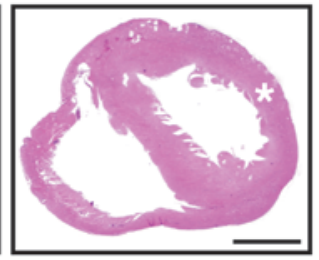

D

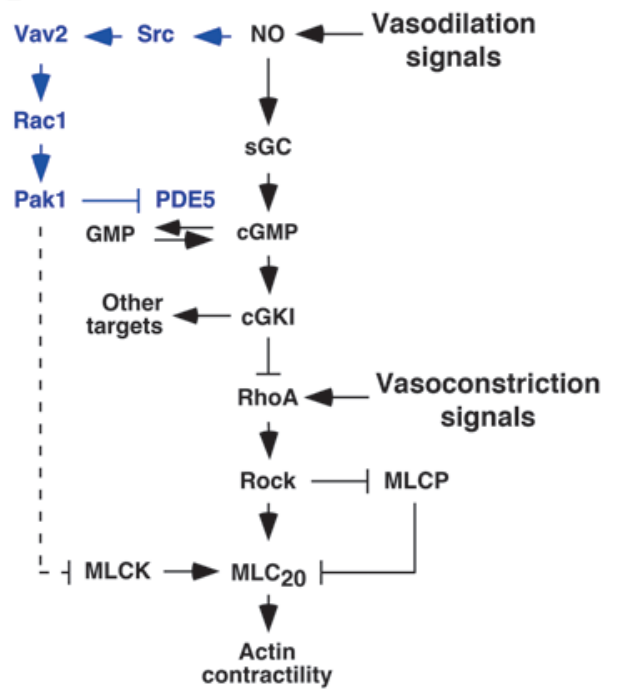

Figure 11

Blood pressure and cardiovascular parameters in sildenafil-treated Vav2 $^{-1-}$ mice. (A) Arterial pressure (left panel $n=5-9$ ), heart rates (middle panel $n=5-9$ ), and plasma levels of angiotensin II (right panel $n=5-9$ ) in 4-month-old mice of the indicated genotypes that were either untreated $(-)$ or treated $(+)$ with sildenafil for 4 months. ${ }^{\#} P<0.05$; ${ }^{*} P<0.01$ compared with wild-type controls. (B) Histological sections of aortas (2 left columns) and hearts (2 right columns) of 4-month-old mice of the indicated genotypes, which were previously left untreated $(-)$ or treated $(+)$ with sildenafil for 4 months as indicated. Asterisks mark the aortic media walls and the left heart ventricles. Sections are representative samples of 5 mice of each genotype. Scale bars: $100 \mu \mathrm{m}$ (arteries); $1 \mathrm{~mm}$ (hearts). (C) Size of the aorta media wall (upper panel) and myocytes (lower panel) from 4-monthold animals of the indicated genotypes treated $(+)$ or untreated $(-)$ with sildenafil for 4 months $(n=5)$. ${ }^{\star} P<0.01$ compared with wild-type controls. Data are shown as mean + SEM. (D) Schematic representation of the new pathway reported in this work (blue color). The previously known signaling elements and connections of the NO signaling route in VSMCs are shown in black. Activation steps are indicated by arrows. Inhibitory steps are indicated by blunted lines. Putative connections among molecules are indicated as broken lines.
It has been recently shown that Rac1 and Pak 1 can regulate cGMP production in other cell types by stimulating transmembrane GCs (41). However, in the case of vSMCs, the overall levels of cGMP are regulated by the combined action of cytosolic sGC and PDE5 rather than by membrane-bound GCs (9). Unlike the case of its transmembrane counterparts, $\mathrm{SGC}$ is activated in cis by the direct binding of $\mathrm{NO}$ to their prosthetic heme group rather than by intracellular routes in trans. Consistent with this, we have shown that Pak1 does not affect the basal and NO-induced enzyme activities of sGC, a result that agrees with prior observa- tions made with Rac1 in vivo (41). Likewise, using specific agonists and inhibitors of the $\mathrm{NO} / \mathrm{sGC} / \mathrm{cGKI}$ route, we have demonstrated that the signaling defects of primary $\operatorname{Vav}^{-{ }^{--}}$vSMCs, Vav2 $2^{-/-}$aortas, and $\operatorname{Vav} 2^{-/-}$renal arteries derive from excessive levels of cGMP catabolism rather than from ineffective synthesis of this second messenger. These results indicate that PDE5 is the only target of the Vav2/Rac1/Pak1 route in vSMCs and, in addition, that Pak1 may affect the intracellular levels of cGMP through different signaling mechanisms, depending on the cell type and stimulation conditions involved. 
The physiological importance of the new signaling route reported here is demonstrated by the reactivity problems found in the blood vessels of Vav2 $2^{-1-}$ mice toward NO-dependent vasodilation stimuli, the deficient production of cGMP upon acetylcholine stimulation of $\mathrm{Vav}^{-/-}$aortas, and the rescue of normal vasodilatation responses using PDE5 inhibitors (zaprinast) or cGMP analogues. Furthermore, the observations indicating that the chronic pharmacological inactivation of PDE5 prevents the development of hypertension and cardiovascular disease in Vav2 $2^{-/-}$mice clearly highlight the contribution of this route to normotensia maintenance in vivo. It should be noted, however, that Vav2 $2^{-/-}$mice may have additional problems that could contribute to the development of their hypertensive state. On the one hand, the vasodilatation problems induced by the abnormal regulation of PDE5 reported here can in turn trigger the stimulation of downstream physiological circuits that can cooperate in the development of hypertension by promoting vasoconstriction and in the establishment of hypertension-associated pathologies such as cardiovascular remodeling and tissue fibrosis. These downstream events may synergize among themselves to provide even stronger vasoconstriction signals, as previously demonstrated by the detection of a positive crosstalk between renin/angiotensin II and noradrenaline secretion in Vav2 $2^{-/}$mice (24). This functional scenario can explain the marked antihypertensive and antivascular remodeling effects of drugs that block angiotensin II biosynthesis (captopril) and angiotensin II signaling (losartan) in those animals (24). Consistent with an upstream position of the PDE5 regulatory defect relative to the aforementioned physiological circuits, we have observed that the chronic administration of sildenafil blocks the elevation of the plasma levels of angiotensin II and noradrenaline in $\mathrm{Vav}^{-2^{--}}$mice. On the other hand, it is likely that $\mathrm{Vav}^{2^{-/}}$mice could have alterations in other systems involved in cardiovascular regulation (i.e., the autonomic nervous system) that also contribute to the establishment of their hypertensive state. The latter possibility is supported by the observation that $\mathrm{Vav}^{2^{-/}}$mice are tachycardic and display a hypertrophy of the adrenal gland medulla (24), 2 dysfunctions that are not eliminated by sildenafil. Furthermore, sildenafil has only a partial inhibitory effect on the elevated plasma levels of adrenaline, aldosterone, and vasopressin present in Vav2-deficient mice. The cause of these additional defects remains to be identified. It is likely, however, that they are mediated by sympathetic events, since the addition of a $\beta$-adrenergic receptor antagonist (propranolol) totally eliminates the high levels of vasopressin and aldosterone present in these mice (24). Whereas it is likely that these additional defects also contribute to the development of the hypertensive state of $\mathrm{Vav}^{-/-}$mice (24), the marked antihypertensive effects of the sildenafil treatments clearly indicate that the hyperactivity of PDE5 is a major contributor, rather than a contingent one, to the hypertensive condition and cardiovascular disease found in $\operatorname{Vav}^{2^{-/}}$mice. Moreover, it is clear that the sympathetic component of the cardiovascular phenotype that is not responsive to the sildenafil treatments cannot trigger per se hypertension and cardiovascular remodeling because Vav2 $2^{-/-}$mice subjected to long-term treatments with sildenafil do not develop hypertension and renocardiovascular disease despite the fact that they still have moderate levels of adrenaline and renalrelated hormones in their plasma.

Interestingly, we have not detected any reactivity problems to NO in blood vessels and primary vSMCs from Vav3-deficient mice. Thus, we have observed that the sildenafil treatment has no effects on the evolution of the hypertensive condition of $\mathrm{Vav3}^{-/-}$mice, indicating that the beneficial action of this drug on the cardiovascular phenotype of $\mathrm{Vav}^{-/-}$mice is a reflection of the intrinsic signaling defects present in Vav2 $2^{-/}$mice. These results also suggest that the defects causing the loss of normotensia in these 2 knockout mouse models are not identical. Consistent with this view, we have previously described those 2 mouse strains as differing in a number of physiological parameters. For example, Vav3 $3^{-/}$mice, but not Vav2 $2^{-/-}$mice, display high levels of dopamine in plasma $(24,25)$. Furthermore, and consistent with what we have observed with the long-term sildenafil treatments in this work, we have previously shown that captopril eliminates the elevated noradrenaline plasma levels in Vav2-deficient mice but not in $\mathrm{Vav3}^{-/-}$animals $(24,25)$. Finally, Vav3 $3^{-/-}$, but not Vav2 $2^{-/}$, mice show tachypnea (V. Sauzeau and X.R. Bustelo, unpublished observations). In this context, recent data obtained in our laboratory suggest that the hypertension in $\mathrm{Vav}^{-3^{--}}$mice probably derives from axon guidance defects in GABAergic neurons located in the ventrolateral medulla (V. Sauzeau and X.R. Bustelo, manuscript in preparation), a brainstem region that plays key roles in the control of blood pressure, heart, and breathing rates in mammals (42). Interestingly, the double Vav2 $2^{-/}$; Vav3 $^{-/-}$animals show a combination of the phenotypes of the single-knockout mice (24) (this paper and data not shown), although their total values of blood pressure and kinetics of cardiovascular remodeling are not significantly aggravated when compared with Vav2 ${ }^{-I^{-}}$and $\operatorname{Vav}^{-{ }^{--}}$mice (24).

It has been previously shown that Rac1 plays important roles in the regulation of eNOS activity in endothelial cells. For example, a recent report using tissue-specific knockin mice with a $50 \%$ reduction of Rac1 in the endothelium has revealed that this GTPase regulates eNOS function at several levels, including the upregulation of ENOS gene transcription, ENOS transcript stability, and eNOS catalytic activity. As a consequence, these mice display reduced NO bioavailability and a mild hypertension (43). Some of these responses have been reported to be Pak1 dependent (43). Rac1 has been also linked to the activation of eNOS in endothelial cells mediated by both sphingosine 1-phosphate and AMP-activated protein kinase $(44,45)$. Although the Rac1 GEFs that mediate these effects are hitherto unknown, it has been proposed that the sphingosine 1-phosphate-mediated activation of Rac1 is mediated by the Tiam 1 GEF (44). The new signal transduction pathway identified in the present work in vSMCs suggests that the Rac1 route is utilized in a concerted and stepwise manner by endothelial and vascular smooth muscle cells to ensure optimal, NO-dependent vasodilatation responses. Thus, whereas the stimulation of the Rac1 pathway in endothelial cells makes the production of optimal amounts of $\mathrm{NO}$ possible, the subsequent activation of this GTPase in NO-stimulated vSMCs favors the establishment of robust downstream vasodilation responses by ensuring the inhibition of the cGMP-hydrolyzing activity of PDE5 during the earlier signaling responses to this gas.

Although the cardiovascular phenotype of $\mathrm{Vav}^{-/-}$mice led us to focus our analysis exclusively on vSMCs and the F-actin cytoskeleton, it is worth noting that the functional role of the Rac1/ Pak1 pathway reported here could potentially be more complex both in terms of cell types and biological activities affected. For example, PDE5 has been linked to myocyte contraction and relaxation, erectile function, platelet aggregation, and a number of pathophysiological processes $(16,17)$. Furthermore, mammalian tissues contain 10 additional PDE family members and many 
isoforms specific for cGMP, cAMP, or both cGMP and cAMP (12). Interestingly, 5 of those family members (PDE2, PDE6, PDE10A1, PDE10A2, PDE11A4) contain N-terminal regions very similar in structure to the Pak1-docking platform identified on PDE5. These PDE5-like proteins affect a number of cellular functions, including photoreceptor cell survival, memory and locomotor activity, and spermatogenesis (12). Thus, although is clear that the phenotype of Vav2 $2^{-/}$vSMCs is mediated by the defective regulation of the PDE5 isoform, it is tempting to speculate that the Rac1/Pak1 route, via upstream regulation by either Vav2 or other Rac1-specific GDP/ GTP exchange factors, may potentially modulate the intracellular levels of cyclic nucleotide monophosphates in other cell types, biological responses, and pathologies by targeting either PDE5 or structurally related PDE family members. The severe impact of the Vav2/Rac1 pathway on vSMC cGMP levels also indicates that this route may participate in signaling diversification and amplification events by favoring the stimulation of additional pathways lying downstream of this second messenger and/or cGKI.

Some inferences from the present work also deserve further investigation in the future. Thus, the observation that NO induces Vav2 phosphorylation suggests that the activation of Vav family proteins can be achieved by mechanisms different from the canonical stimulation by transmembrane protein receptors (19). Since independent reports have shown that NO and Vav proteins play important roles in T lymphocytes $(46,47)$, it will be interesting to investigate whether these 2 molecules could also work in a concerted manner in lymphocytes or other cell types. Finally, the critical role of this new pathway in NO-mediated vasodilatation suggests that the development of agonists for its components may represent a potential pharmacological avenue in the future to treat cardiovascular diseases caused by defective blood vessel reactivity to NO.

\section{Methods}

Animals. Mouse strains used in this work have been reported before $(24,25$, $48,49)$ and homogenized in a C57BL/6 background. Vav $2^{-/-}$and $\mathrm{RhoG}^{-/-}$mice were provided by M. Turner (Laboratory of Lymphocyte Signaling and Development, The Babraham Institute, Cambridge, United Kingdom). All animal studies were approved by the Bioethics Committees of both the University of Salamanca and the Spanish Research Council (Madrid, Spain). Unless otherwise indicated, we used 4-month-old animals for the experiments reported in this work. In the case of single-knockout animals, we always included animals derived from crosses of heterozygous mice. In the case of double-knockout animals, we used litters derived from independent crosses of wild-type and $\operatorname{Vav}_{2-/-;} \operatorname{Vav}^{-/-}$mice that were born at the same time.

Determination of blood vessel reactivity levels. Mice were anesthetized with an intraperitoneal injection of sodium pentobarbital ( $40 \mathrm{mg} / \mathrm{kg}$ body weight) (Sigma-Aldrich). After an abdominal incision, the right kidney was isolated and the right renal artery cannulated and perfused at $1 \mathrm{ml} / \mathrm{min}$ with a Krebs-Henseleit solution ( $118.4 \mathrm{mM} \mathrm{NaCl}, 4.7 \mathrm{mM} \mathrm{KCl}, 2 \mathrm{mM} \mathrm{CaCl}_{2}, 1.2$ $\mathrm{mM} \mathrm{MgSO}_{4}, 1.2 \mathrm{mM} \mathrm{KH}_{2} \mathrm{PO}_{4}, 25 \mathrm{mM} \mathrm{NaHCO}_{3}$ and $11 \mathrm{mM}$ glucose). The buffer was maintained at $37^{\circ} \mathrm{C}$ in a $95 \% \mathrm{O}_{2} / 5 \% \mathrm{CO}_{2}$ atmosphere. After a 60 -minute equilibration period, arteries were perfused with $1 \mu \mathrm{M}$ phenylephrine (Sigma-Aldrich) and then with graded doses $(0.01$ to $100 \mu \mathrm{M})$ of Ach (Sigma-Aldrich), SNP (Sigma-Aldrich), or isoproterenol (Sigma-Aldrich) at the indicated times. In the case of 1-month-old mice, SNP gradient doses ranged from $0.1 \mathrm{nM}$ to $1 \mu \mathrm{M}$. When indicated, vasodilatation was induced by graded doses of 8-pCPT-cGMP (Biolog). Arterial contractility was induced by perfusing renal arteries with either $60 \mathrm{mM} \mathrm{KCl}$ or $0.1 \mu \mathrm{M}$ angiotensin II (Sigma-Aldrich). Renal perfusion pressures were monitored with a pressure probe linked to a digital data recorder (MacLab/4e; $\mathrm{AD}$ Instruments).
Recordings were analyzed using Chart v3.4 software (AD Instruments). In the case of aortas, 2-mm-long aorta rings obtained from mice of the indicated genotypes were cut and mounted onto a 4-channel myograph (Danish Myo Technology) under isometric techniques. Each vessel was then normalized to determine maximum active tension development. Contraction measurements were performed under isometric conditions in Krebs-Henseleit solution as above. Each channel was connected to a force transducer to measure contraction and relaxation responses with the indicated stimuli.

Preparation of primary aortic smooth muscle cell cultures. Aortas were collected from the indicated mouse strains and cleaned manually to remove fat, adherent connective tissue, and endothelial cells. Cleaned aortas were cut into approximately $5 \times 5-\mathrm{mm}$ pieces and cultured in DMEM (Gibco; Invitrogen) supplemented with $10 \%$ fetal calf serum (Gibco; Invitrogen) and antibiotics (Gibco; Invitrogen) at $37^{\circ} \mathrm{C}$ in a $5 \% \mathrm{CO}_{2}$ atmosphere. The culture medium was changed every 48 hours. After approximately 2 weeks, the vSMCs populating the plate were trypsinized and reseeded in fresh DMEM supplemented with $10 \%$ fetal calf serum and antibiotics. This stage of the vSMC culture was considered as passage 1. One passage more was done by trypsinization of confluent cultures of vSMCs to obtain passage 2 . Only vSMCs at passage 2 were used in these studies.

Analysis of F-actin cytoskeletal dynamics. Isolated primary vSMCs were fixed with $4 \%$ formaldehyde in PBS for 20 minutes and then permeabilized for 10 minutes in PBS containing 0.5\% Triton X-100 (Sigma-Aldrich). For F-actin staining, cells were simultaneously incubated in some cases with 2 units $/ \mathrm{ml}$ of rhodamine-conjugated phalloidin (Molecular Probes; Invitrogen) and 50 $\mu \mathrm{g} / \mathrm{ml}$ of Alexa Fluor 488-labeled DNaseI (Molecular Probes; Invitrogen) at room temperature for 30 minutes to visualize F-actin and monomeric G-actin, respectively. In other cases (i.e., retroviral-infected cultures expressing GFP), cells were only incubated with rhodamine-conjugated phalloidin as indicated above. After staining, images were captured using a fluorescence microscope (Zeiss) and quantified. To make side-by-side comparisons possible, we captured fluorescent signals, always keeping constant the time of image capturing and the image intensity gain at both wavelengths. Quantitation of fluorescence signals was done using Metamorph/MetaView software (Universal Imaging). To this end, we first subtracted electronically the background fluorescence signal present in cell-free areas of the captured images and, subsequently, measured the rhodamine- and, when appropriate, Alexa Fluor 488-derived fluorescence signals of selected cells. When using double F-actin/G-actin labeling, the ratio of fluorescence of rhodamine-phalloidin and Alexa Fluor 488-DNaseI (F-actin/G-actin ratio) was used to quantify the actin cytoskeleton organization, always assigning an arbitrary value of 1 to the signal obtained in the respective wild-type vSMC control. When using single F-actin staining, the fluorescence of rhodaminephalloidin was used, assigning as before an arbitrary value of 1 to the signals obtained in the wild-type control samples. In all cases, we utilized a minimum of 50 single, randomly picked cells (in the case of experiments involving nontransfected cells) or 50 GFP-positive cells (in the case of retrovirally infected cells; see below) in each experimental condition.

Pharmacological treatments in primary vascular smooth muscle cells. SNP (10 $\mu \mathrm{M}$; Sigma-Aldrich) was used for the indicated periods of time. When not indicated, the stimulation with this NO donor lasted 1 hour. PP2 and PP3 (10 $\mu \mathrm{M}$ each; Calbiochem) were added to cultures 1 hour before SNP stimulation. BAY 41-2272 (1 $\mu \mathrm{M}$; Alexis Biochemicals), 8-Br-cGMP (50 $\mu \mathrm{M}$; Bio$\log )$, and Y-27632 (10 $\mu \mathrm{M}$; Calbiochem) were added to cultures for 1 hour. 1-(5-iodonaphthalene-1-sulfonyl)-1H-hexahydro-1,4-diazepine (1 $\mu \mathrm{M}$; Sigma-Aldrich) was added to the vSMC cultures for 30 minutes. When indicated, cells were pretreated with zaprinast (10 $\mu \mathrm{M}$; Tocris), vinpocetine (10 $\mu \mathrm{M}$; Sigma-Aldrich), or EHNA hydrochloride (10 $\mu \mathrm{M}$; Sigma-Aldrich) for 1 hour before addition of agonists. Tat-Pak18 and Tat-Pak ${ }^{\mathrm{R} 192 \mathrm{~A}}$ (10 $\mu \mathrm{M}$ each; Calbiochem) were added to cultures 12 hours before SNP stimulation. 
Immunoblots, immunoprecipitations, and pulldown experiments. Isolation and detection of proteins in cytosolic and membrane-enriched fractions was done as described previously (10). Immunoprecipitation and pulldown experiments were performed according to standard techniques (see Supplemental Methods for a detailed description of protocols, immunoreagents, and GST fusion proteins used).

cGMP concentration determination. We used an ELISA kit (Cyclic GMP EIA Kit; Cayman Chemical) according to the supplier's specifications to measure intracellular cGMP concentration in vSMCs.

PDE5 activity assays. PDE5 enzyme activity was measured using the IMAP Phosphodiesterase Assay Kit (Molecular Devices) according to the manufacturer's protocol. In brief, 50 units/ $\mu$ l of PDE5 (Calbiochem) in either the absence or presence of $150 \mathrm{ng}$ of purified GST-Pak1 protein (Invitrogen) was incubated for 1 hour at $30^{\circ} \mathrm{C}$ in $20 \mathrm{mM}$ Tris- $\mathrm{HCl}(\mathrm{pH} 8.0), 10 \mathrm{mM}$ $\mathrm{MgCl}_{2}$, and $1 \mathrm{mM}$ DTT either with or without $100 \mu \mathrm{M}$ ATP. Samples were then diluted to obtain an enzyme dilution curve ( 0.39 to 50 units $/ \mathrm{ml}$ ) with 3 replicates. Activity of samples was finally measured by the stepwise room temperature incubations with the cGMP substrate solution for 45 minutes and then the IMAP binding reagent for 30 minutes. Final reaction products were measured using a microplate reader (Ultra Evolution; Tecan).

In vitro kinase reactions. $1 \mu \mathrm{g}$ of either GST-PDE5 fusion proteins purified from E. coli (see Supplemental Methods) or histone 1 (Sigma-Aldrich) was incubated with $150 \mathrm{ng}$ of GST-Pak1, cGKIa (Promega), or PKA (Promega) in $50 \mu \mathrm{l}$ of a solution containing $20 \mathrm{mM}$ Tris- $\mathrm{HCl}$ ( $\mathrm{pH} 8.0$ ), $10 \mathrm{mM} \mathrm{MgCl} 2,1 \mathrm{mM}$ DTT, $100 \mu \mathrm{M}$ ATP, and $1 \mu \mathrm{Ci}[32 \gamma$-P] ATP (Amersham Biosciences; GE Healthcare) for 1 hour at $30^{\circ} \mathrm{C}$. Reactions were stopped by adding $2 \times$ SDS-PAGE sample buffer. SDS-PAGE gels with the electrophoretically separated proteins were fixed, dried, and subjected to autoradiography at $-70^{\circ} \mathrm{C}$.

1. Bustelo XR, Sauzeau V, Berenjeno IM. GTPbinding proteins of the Rho/Rac family: regulation, effectors and functions in vivo. Bioessays. 2007;29(4):356-370.

2. Etienne-Manneville S, Hall A. Rho GTPases in cell biology. Nature. 2002;420(6916):629-635.

3. Jaffe AB, Hall A. Rho GTPases: biochemistry and biology. Annu Rev Cell Dev Biol. 2005;21:247-269.

4. Budzyn K, Marley PD, Sobey CG. Targeting Rho and Rho-kinase in the treatment of cardiovascular disease. Trends Pharmacol Sci. 2006;27(2):97-104.

5. Amano $\mathrm{M}$, et al. Phosphorylation and activation of myosin by Rho-associated kinase (Rho-kinase). J Biol Chem. 1996;271(34):20246-20249.

6. Kimura K, et al. Regulation of myosin phosphatase by Rho and Rho-associated kinase (Rho-kinase). Science. 1996;273(5272):245-248.

7. Mueller BK, Mack H, Teusch N. Rho kinase, a promising drug target for neurological disorders. Nat Rev Drug Discov. 2005;4(5):387-398.

8. Hofmann F, Feil R, Kleppisch T, Schlossmann J. Function of cGMP-dependent protein kinases as revealed by gene deletion. Physiol Rev. 2006;86(1):1-23.

9. Gong L, Pitari GM, Schulz S, Waldman SA. Nitric oxide signaling: systems integration of oxygen balance in defense of cell integrity. Curr Opin Hematol. 2004;11(1):7-14.

10. Sauzeau V, et al. Cyclic GMP-dependent protein kinase signaling pathway inhibits RhoA-induced $\mathrm{Ca} 2+$ sensitization of contraction in vascular smooth muscle. J Biol Chem. 2000;275(28):21722-21729.

11. Hirano K, Derkach DN, Hirano M, Nishimura J, Kanaide H. Protein kinase network in the regulation of phosphorylation and dephosphorylation of smooth muscle myosin light chain. Mol Cell Biochem. 2003;248(1-2):105-114.

12. Omori K, Kotera J. Overview of PDEs and their regulation. Circ Res. 2007;100(3):309-327.

13. Huang PL, et al. Hypertension in mice lacking the
Inbibition of PDE5 in vivo. Sildenafil (20 mg/ $\mathrm{kg}$ body weight/d; Pfizer) was added in drinking water to 1-month-old wild-type, Vav2 $2^{-/}$, and Vav3 $3^{-/-}$ mice. The analyses of cardiovascular parameters, angiotensin II levels, and histological analyses were carried out after a 3-month-long treatment as indicated $(24,25)$.

Statistics. Data were analyzed using either the 2-tailed Student's $t$ test or the 1 -way ANOVA test. Data are given as the mean \pm SEM. $P$ values lower than 0.05 were considered statistically significant.

\section{Acknowledgments}

We thank M. Blázquez, T. Iglesias, and M. Menacho-Márquez for technical help, M. Turner for Vav2 $2^{-/-}$and $R h o G^{-/-}$mice, and M. Dosil for helpful comments on the manuscript. X.R. Bustelo's work is supported by grants from the NIH (5R01CA73735), the Spanish Ministry of Science and Innovation (MSI) (SAF200601789), the Red Temática de Investigación Cooperativa en Cáncer (RD06/0020/0001), and the Castilla y León Autonomous Government (SA053A05 and GR97). V. Sauzeau was partially supported by a European Molecular Biology Organization (EMBO) long-term postdoctoral fellowship, an MSI Juan de la Cierva postdoctoral contract, and currently, an MSI/CSIC Ramón y Cajal contract.

Received for publication December 16, 2008, and accepted in revised form October 14, 2009.

Address correspondence to: Xosé R. Bustelo, Centro de Investigación del Cáncer, CSIC-University of Salamanca, Campus Unamuno, E-37007 Salamanca, Spain. Phone: 34-923294802; Fax: 34923294743; E-mail: xbustelo@usal.es. gene for endothelial nitric oxide synthase. Nature. 1995;377(6546):239-242.

14. Pfeifer A, et al. Defective smooth muscle regulation in cGMP kinase I-deficient mice. EMBO J. 1998;17(11):3045-3051.

15. Shesely EG, et al. Elevated blood pressures in mice lacking endothelial nitric oxide synthase. Proc Natl Acad Sci U S A. 1996;93(23):13176-13181.

16. Kass DA, Takimoto E, Nagayama T, Champion HC. Phosphodiesterase regulation of nitric oxide signaling. Cardiovasc Res. 2007;75(2):303-314.

17. Ravipati G, McClung JA, Aronow WS, Peterson SJ, Frishman WH. Type 5 phosphodiesterase inhibitors in the treatment of erectile dysfunction and cardiovascular disease. Cardiol Rev. 2007;15(2):76-86.

18. Guilluy C, et al. Inhibition of RhoA/Rho kinase pathway is involved in the beneficial effect of sildenafil on pulmonary hypertension. Br J Pharmacol. 2005;146(7):1010-1018.

19. Bustelo XR. Regulatory and signaling properties of the Vav family. Mol Cell Biol. 2000;20(5):1461-1477.

20. Bustelo XR. Vav2 [published online March 7, 2008]. UCSD - Nature Molecule Pages. doi:10.1038/ mp.a002361.01.

21. Bustelo X, Couceiro JR. Vav3 [published online March 7, 2008]. UCSD - Nature Molecule Pages. doi:10.1038/mp.a002362.01.

22. Movilla N, Bustelo XR. Biological and regulatory properties of Vav-3, a new member of the Vav family of oncoproteins. Mol Cell Biol. 1999; 19(11):7870-7885.

23. Schuebel KE, et al. Isolation and characterization of murine vav2, a member of the vav family of proto-oncogenes. Oncogene. 1996;13(2):363-371.

24. Sauzeau V, Jerkic M, Lopez-Novoa JM, Bustelo XR. Loss of Vav2 proto-oncogene causes tachycardia and cardiovascular disease in mice. Mol Biol Cell. 2007;18(3):943-952.

25. Sauzeau V, et al. Vav3 proto-oncogene deficiency leads to sympathetic hyperactivity and cardiovascular dysfunction. Nat Med. 2006;12(7):841-845.

26. Furchgott RF, Zawadzki JV. The obligatory role of endothelial cells in the relaxation of arterial smooth muscle by acetylcholine. Nature. 1980; 288(5789):373-376

27. Schuebel KE, Movilla N, Rosa JL, Bustelo XR. Phosphorylation-dependent and constitutive activation of Rho proteins by wild-type and oncogenic Vav-2. EMBO J. 1998;17(22):6608-6621.

28. Akhand AA, et al. Nitric oxide controls src kinase activity through a sulfhydryl group modificationmediated Tyr-527-independent and Tyr-416-linked mechanism. J Biol Chem. 1999;274(36):25821-25826.

29. Abe K, et al. Vav2 is an activator of Cdc42, Rac1, and RhoA. J Biol Chem. 2000;275(14):10141-10149.

30. Guo F, Zheng Y. Rho family GTPases cooperate with p53 deletion to promote primary mouse embryonic fibroblast cell invasion. Oncogene. 2004;23(33):5577-5585.

31. Lin R, Bagrodia S, Cerione R, Manor D. A novel Cdc42Hs mutant induces cellular transformation. Curr Biol. 1997;7(10):794-797.

32. Joneson T, McDonough M, Bar-Sagi D, Van Aelst L. RAC regulation of actin polymerization and proliferation by a pathway distinct from Jun kinase. Science. 1996;274(5291):1374-1376.

33. Maruta $\mathrm{H}, \mathrm{He} H$, Nheu T. Interfering with Ras signaling using membrane-permeable peptides or drugs. Methods Mol Biol. 2002;189:75-85.

34. Frost JA, Khokhlatchev A, Stippec S, White MA, Cobb MH. Differential effects of PAK1-activating mutations reveal activity-dependent and -independent effects on cytoskeletal regulation. J Biol Chem. 1998;273(43):28191-28198.

35. Sells MA, et al. Human p21-activated kinase (Pak1) regulates actin organization in mammalian cells. Curr Biol. 1997;7:202-210.

36. Daniels RH, Hall PS, Bokoch GM. Membrane tar- 
geting of p21-activated kinase 1 (PAK1) induces neurite outgrowth from PC12 cells. EMBOJ. 1998; 17(3):754-764.

37. Higuchi M, Onishi K, Kikuchi C, Gotoh Y. Scaffolding function of PAK in the PDK1-Akt pathway. Nat Cell Biol. 2008;10(11):1356-1364.

38. Bokoch GM. Biology of the p21-activated kinases. Annu Rev Biochem. 2003;72:743-781.

39. Lei M, et al. Structure of PAK1 in an autoinhibited conformation reveals a multistage activation switch. Cell. 2000;102(3):387-397.

40. Parrini MC, Lei M, Harrison SC, Mayer BJ. Pak1 kinase homodimers are autoinhibited in trans and dissociated upon activation by Cdc42 and Rac1. Mol Cell. 2002;9(1):73-83.
41. Guo D, et al. A Rac-cGMP signaling pathway. Cell. 2007;128(2):341-355.

42. Guyenet PG. The sympathetic control of blood pressure. Nat Rev Neurosci. 2006;7(5):335-346.

43. Sawada N, Salomone S, Kim HH, Kwiatkowski DJ, Liao JK. Regulation of endothelial nitric oxide synthase and postnatal angiogenesis by Rac1. Circ Res. 2008;103(4):360-368.

44. Gonzalez E, Kou R, Michel T. Rac1 modulates sphingosine 1-phosphate-mediated activation of phosphoinositide 3-kinase/Akt signaling pathways in vascular endothelial cells. J Biol Chem. 2006; 281(6):3210-3216

45. Levine YC, Li GK, Michel T. Agonist-modulated regulation of AMP-activated protein kinase (AMPK) in endothelial cells. Evidence for an AMPK -> Rac1 -> Akt -> endothelial nitric-oxide synthase pathway. J Biol Chem. 2007;282(28):20351-20364.

46. Tybulewicz VL, Ardouin L, Prisco A, Reynolds LF. Vav1: a key signal transducer downstream of the TCR. Immunol Rev. 2003;192:42-52.

47. Ibiza S, et al. Endothelial nitric oxide synthase regulates $\mathrm{T}$ cell receptor signaling at the immunological synapse. Immunity. 2006;24(6):753-765.

48. Doody GM, et al. Signal transduction through Vav-2 participates in humoral immune responses and $\mathrm{B}$ cell maturation. Nat Immunol. 2001;2(6):542-547.

49. Vigorito E, et al. Immunological function in mice lacking the Rac-related GTPase RhoG. Mol Cell Biol. 2004;24(2):719-729. 